\title{
Nutritional support and dietary interventions for women with polycystic ovary syndrome
}

\author{
This article was published in the following Dove Press journal: \\ Nutrition and Dietary Supplements \\ 6 September 2017 \\ Number of times this article has been viewed
}

\section{Kleopatra Papavasiliou \\ Emilia Papakonstantinou \\ Unit of Human Nutrition, Department of Food Science and Human Nutrition, Agricultural University of Athens, Athens, Greece}

\begin{abstract}
Polycystic ovary syndrome (PCOS) is a common endocrine disorder in reproductiveaged women, which leads to reproductive, metabolic and hormonal abnormalities. Hyperinsulinemia, insulin resistance, androgen excess, ovulatory dysfunction, polycystic ovaries, gonadotropin abnormalities, obesity, adipose tissue dysfunction, difficulty to conceive and high-risk pregnancy are the most common PCOS-associated complications. The aim of this review was to describe and evaluate the effects of dietary interventions on PCOS-associated outcomes and to provide some evidence-based dietary advice for use in clinical practice. There is no optimal diet or macronutrient composition for PCOS. However, lifestyle modification, including a small-to-moderate weight loss of 5-10\% (combined diet with regular physical activity) with any dietary pattern of choice, depending on the individuals' preferences, culture, habits and metabolic needs (ie, Mediterranean diet, Dietary Approaches to Stop Hypertension [DASH] diet or moderately low-carbohydrate diets [30-45\% of energy]), as well as alternative dietary interventions, including small, frequent meal (five to six meals daily) consumption at regular times, with the majority of carbohydrates consumed at lunch time or equally distributed throughout the day, seems to offer the evidence-based first-line strategy for the management of PCOS symptoms and insulin resistance. No conclusions can be drawn at this time for high protein diets, polyunsaturated fatty acids or micronutrient supplementation.
\end{abstract}

Keywords: nutrition, meal frequency, dietary strategies, insulin resistance

\section{Introduction}

Polycystic ovary syndrome (PCOS) is a complex endocrine disorder in reproductiveaged women with a prevalence from $4 \%$ up to $18 \%$, is the most common cause of infertility and is associated with reproductive, metabolic and hormonal dysfunction and higher association of pregnancy complications. ${ }^{1-8}$ Women with PCOS with menstrual irregularities, related to anovulation, may have difficulties to conceive. ${ }^{5}$ The majority of women with PCOS have hyperinsulinemia and insulin resistance (IR), which have a significant role in the pathogenesis of the syndrome and in the long run may lead to impairment of glucose metabolism, androgen excess with hyperandrogenic symptoms, gonadotropin abnormalities, ovulatory dysfunction and polycystic ovaries, higher intra-abdominal fat and adipose tissue dysfunction, independently of body mass index (BMI). ${ }^{5,9-18}$

Women with PCOS usually weigh more than women without PCOS, and many studies show that more than half of women with PCOS are found to be obese and tend to gain more weight longitudinally compared to women without PCOS. ${ }^{5,19-22}$ 
However, despite the fact that the majority of women with PCOS are overweight or obese, many lean women with PCOS are also considered to be at increased risk for metabolic disturbances..$^{23,24}$ Central obesity, race and age are positively associated with IR. ${ }^{14,25,26}$ Central obesity and excess body fat exacerbate IR, dyslipidemia and hormonal dysfunction. ${ }^{5,26-29}$ Moreover, women with PCOS have increased risk of metabolic syndrome, gestational diabetes, type 2 diabetes and cardiovascular diseases. ${ }^{8,22,26,29,31-33}$

The main cause of infertility in women with PCOS is anovulation, whereas obesity seems to exacerbate symptoms and reduces conceiving rates. ${ }^{5,30}$ Some of the mechanisms involved in infertility may be ovarian hyperandrogenism, including luteinizing hormone (LH) hypersecretion, relative follicle-stimulating hormone (FSH) insufficiency, hyperinsulinemia and high anti-Mullerian hormone (AMH) inhibiting aromatase activity. ${ }^{5}$ Some adipokines secreted by adipose tissue may also be involved in obesity-mediated infertility, including low levels of adiponectin, related to androgen excess, and increased visfatin levels, related to glucose dysmetabolism. ${ }^{5,16}$ Leptin, resistin and retinolbinding protein 4 do not seem to play a significant role in PCOS with obesity-related infecundity. ${ }^{5,16}$ However, leptin is positively associated with BMI and LH, but not insulin, in women with PCOS. ${ }^{34}$
Diet and its effect on metabolic outcomes should be more thoroughly examined in women with PCOS. Women with PCOS seem to have a greater appetite, consume more energy-dense high glycemic index (GI) foods and saturated fat, have inadequate fiber intake and have low scores for PCOS-related quality of life, although their overall energy intake, physical activity and resting metabolic rate are similar to controls. ${ }^{21,35-37}$

Reduction in IR has been suggested as the principal goal of PCOS treatment. Lifestyle changes (diet plus physical activity), along with weight loss (5-10\%), are proposed as the first-line strategy for amelioration of IR, ovulatory function and decreased free testosterone levels in women with PCOS. ${ }^{38-45}$ Trunk fat, waist circumference (WC) and BMI are the best predictors of IR in PCOS. ${ }^{46}$ Other dietary interventions, including carbohydrate distribution, meal frequency and timing, adequate intake of n-3 fatty acids and/or vitamin D supplementation, have been suggested to offer some additional benefits for markers of glucose and energy metabolism and reproductive hormonal regulation. In this review, we discuss nutritional support and dietary interventions for women with PCOS and attempt to derive to some evidence based conclusions for use in clinical practice. Tables 1 and 2 summarize the dietary interventions for women with PCOS.

Table I Effects of nutritional support and dietary interventions for women with PCOS

\begin{tabular}{|c|c|c|c|c|c|c|}
\hline Study & $\begin{array}{l}\text { Age (years) } \\
\text { BW category } \\
\left(\mathrm{kg} / \mathrm{m}^{2}\right)\end{array}$ & $\begin{array}{l}\text { Duration } \\
\text { and design } \\
\text { of dietary } \\
\text { intervention }\end{array}$ & $\begin{array}{l}\text { Sample } \\
\text { size }\end{array}$ & $\begin{array}{l}\text { Description } \\
\text { of groups }\end{array}$ & $\begin{array}{l}\text { Dietary } \\
\text { intervention }\end{array}$ & $\begin{array}{l}\text { Clinical outcome } \\
\text { measures }\end{array}$ \\
\hline \multicolumn{7}{|c|}{ Dietary interventions with varying macronutrient composition and dietary patterns } \\
\hline Marzouk and & $17-24$ & 6 months & 60 & Weight loss & Deficit of $500 \mathrm{kcal} / \mathrm{day}$ & Significant reduction: \\
\hline \multirow[t]{6}{*}{ Sayed Ahmed ${ }^{77}$} & $36.0 \pm 4.7 / 35.8 \pm 4.8$ & RCT & & group & I5-20\% PRO & BMI $\left(-2.8 \mathrm{~kg} / \mathrm{m}^{2}\right)$ \\
\hline & & & & & $50-55 \% \mathrm{CHO}$ & WC $(-7.8 \mathrm{~cm})$ \\
\hline & & & & & $30 \%$ FAT & Hirsutism (modified FG \\
\hline & & & & Control group & I5-20\% PRO & score, -4.2$)$ \\
\hline & & & & & $50-55 \% \mathrm{CHO}$ & \\
\hline & & & & & $30 \%$ FAT & \\
\hline \multirow[t]{10}{*}{ Wong et $\mathrm{al}^{86}$} & $15.4 \pm 1.3 / 16.3 \pm 2.2$ & 6 months & 16 & LGL diet & $20 \%$ PRO & Decreased in both groups: \\
\hline & $36.2 \pm 5.3 / 33.9 \pm 4.7$ & $\mathrm{RCT}$ & & & $45 \% \mathrm{CHO}$ & BMI percentile $(-I .7 \pm 0.7)$ \\
\hline & & & & & $35 \%$ FAT & Body fat percentage \\
\hline & & & & & low-Gl sources of & $(-1.0 \pm 0.7 \%)$ \\
\hline & & & & & carbohydrates & Trunk fat $(-1.1 \pm 0.7 \mathrm{~kg})$ \\
\hline & & & & LF diet & $20 \%$ PRO & The decrease in BMI \\
\hline & & & & & $55 \% \mathrm{CHO}$ & percentile was greater for \\
\hline & & & & & $25 \%$ FAT & the LF group $(-2.0 \pm 0.6)$ \\
\hline & & & & & & compared to LGL group \\
\hline & & & & & & $(-0.4 \pm 0.1)$ \\
\hline
\end{tabular}

(Continued) 
Table I (Continued)

\begin{tabular}{|c|c|c|c|c|c|c|}
\hline Study & $\begin{array}{l}\text { Age (years) } \\
\text { BW category } \\
\left(\mathrm{kg} / \mathrm{m}^{2}\right)\end{array}$ & $\begin{array}{l}\text { Duration } \\
\text { and design } \\
\text { of dietary } \\
\text { intervention }\end{array}$ & $\begin{array}{l}\text { Sample } \\
\text { size }\end{array}$ & $\begin{array}{l}\text { Description } \\
\text { of groups }\end{array}$ & $\begin{array}{l}\text { Dietary } \\
\text { intervention }\end{array}$ & $\begin{array}{l}\text { Clinical outcome } \\
\text { measures }\end{array}$ \\
\hline Barr et al ${ }^{95}$ & $\begin{array}{l}31.5 \pm 6.9 \\
29.0 \pm 5.9\end{array}$ & $\begin{array}{l}6 \text { weeks } \\
\text { Nonrandomized } \\
\text { clinical trial }\end{array}$ & 21 & $\begin{array}{l}\text { Control phase } \\
\text { (weeks 0-I2) } \\
\text { LGI dietary } \\
\text { intervention } \\
\text { phase (weeks } \\
\text { I2-24) } \\
\text { Follow-up } \\
\text { phase (weeks } \\
\text { 24-36) }\end{array}$ & $\begin{array}{l}\text { Following their } \\
\text { habitual diet } \\
\text { Replace high-GI and } \\
\text { medium-GI foods } \\
\text { with LGI foods } \\
\text { No dietary } \\
\text { intervention } \\
\text { or support }\end{array}$ & $\begin{array}{l}\text { The LGI diet resulted in: } \\
\text { Increased insulin sensitivity } \\
\text { Decreased NEFAs } \\
\text { Small reduction in HDL }\end{array}$ \\
\hline Mehrabani et al ${ }^{83}$ & $\begin{array}{l}28.5 \pm 5.2 / 30.5 \pm 6.4 \\
31 . I \pm 4.6 / 31.9 \pm 4.0\end{array}$ & $\begin{array}{l}3 \text { months } \\
\text { RCT }\end{array}$ & 49 & MHCD & $\begin{array}{l}\text { Deficit of } 500- \\
1000 \text { kcal } \\
\text { I5\% PRO } \\
55 \% \text { CHO } \\
30 \% \text { FAT } \\
\text { Deficit of } 500- \\
1000 \text { kcal } \\
40 \% \text { CHO low and } \\
\text { medium GL } \\
30 \% \text { PRO } \\
30 \% \text { FAT } \\
\text { Limitation of high } \\
\text { glycemic foods }\end{array}$ & $\begin{array}{l}\text { Both hypocaloric diets led } \\
\text { to equal changes in: } \\
\text { BW (CHCD: }-3.3 \pm 0.62 \% \text {, } \\
\text { MHCD: }-4 . I \pm 0.58 \%) \\
\text { LDL-C (CHCD: } \\
-23.7 \pm 13.8 \%, \text { MHCD: } \\
25.5 \pm I 0.5 \%) \\
\text { DHEAS (CHCD: }-32.0 \pm 8.9 \\
\text { ng/mL, MHCD: }-42 . I \pm 16 . I \\
\text { ng/mL) } \\
\text { SHBG (CHCD: } 10.6 \pm 4.1 \\
\text { nmol/L, MHCD: } 8.8 \pm 2.8 \\
\text { nmol/L) } \\
\text { MHCD induced } \\
\text { concomitant reductions in: } \\
\text { Insulin (-3.6 } \pm 0.7 \mathrm{mIU} / \mathrm{L}) \\
\text { HOMA-IR }(-0.8 \pm 0.2) \\
\text { hsCRP (-0.9 } \pm 0.4 \mathrm{mg} / \mathrm{L})\end{array}$ \\
\hline Marsh et $\mathrm{al}^{79}$ & $\begin{array}{l}31.0 \pm 0.7 / 29.3 \pm 0.8 \\
34.3 \pm 1.0 / 34.7 \pm 0.9\end{array}$ & $\begin{array}{l}12 \text { months } \\
\text { or until they } \\
\text { achieved a } 7 \% \\
\text { weight loss }\end{array}$ & 96 & $\begin{array}{l}\text { LF, LGI diet } \\
\text { LF CHD }\end{array}$ & $\begin{array}{l}23 \% \text { PRO } \\
50 \% \text { CHO } \\
27 \% \text { FAT } \\
23 \% \text { PRO } \\
50 \% \text { CHO } \\
27 \% \text { FAT }\end{array}$ & $\begin{array}{l}\text { LGI diet improved post- } \\
\text { OGTT insulin sensitivity } \\
\text { Subjects on LGI diet who } \\
\text { were prescribed metformin } \\
\text { therapy achieved greater } \\
\text { improvements in insulin } \\
\text { sensitivity compared to } \\
\text { those on LGI diet not on } \\
\text { metformin } \\
\text { More women who } \\
\text { consumed the LGI } \\
\text { diet showed improved } \\
\text { menstrual cyclicity ( } 95 \% \\
\text { compared to } 63 \%) \\
\text { Serum fibrinogen } \\
\text { concentrations showed } \\
\text { significant differences } \\
\text { between diets (LGI: } \\
-0.2 \pm 0.1 \text { g/L compared to } \\
\text { CHD: } 0.2 \pm 0.1 \text { g/L) }\end{array}$ \\
\hline
\end{tabular}


Table I (Continued)

\begin{tabular}{|c|c|c|c|c|c|c|}
\hline Study & $\begin{array}{l}\text { Age (years) } \\
\text { BW category } \\
\left(\mathrm{kg} / \mathrm{m}^{2}\right)\end{array}$ & $\begin{array}{l}\text { Duration } \\
\text { and design } \\
\text { of dietary } \\
\text { intervention }\end{array}$ & $\begin{array}{l}\text { Sample } \\
\text { size }\end{array}$ & $\begin{array}{l}\text { Description } \\
\text { of groups }\end{array}$ & $\begin{array}{l}\text { Dietary } \\
\text { intervention }\end{array}$ & $\begin{array}{l}\text { Clinical outcome } \\
\text { measures }\end{array}$ \\
\hline Sørensen et a ${ }^{87}$ & $\begin{array}{l}27.7 \pm 5.5 / 28.4 \pm 5.8 \\
30.6 \pm 7.8 / 30.5 \pm 8.5\end{array}$ & $\begin{array}{l}6 \text { months } \\
\text { RCT }\end{array}$ & 27 & $\begin{array}{l}\text { HP diet } \\
\text { SP diet }\end{array}$ & $\begin{array}{l}>40 \% \text { PRO } \\
<30 \% \text { CHO } \\
30 \% \text { FAT } \\
\text { Ad libitum diet } \\
<15 \% \text { PRO } \\
>55 \% \text { CHO } \\
30 \% \text { FAT } \\
\text { Ad libitum diet }\end{array}$ & $\begin{array}{l}\text { Compared to SP, HP } \\
\text { resulted in significantly } \\
\text { greater decreases in: } \\
\text { BW }(-4.4 \mathrm{~kg}) \\
\text { Fat mass }(-4.3 \mathrm{~kg}) \\
\text { WC }(-3.7 \mathrm{~cm}) \text {, but both } \\
\text { diets equally decreased } \\
\text { Glucose }(\mathrm{HP:} 5.2 \mathrm{mmol} / \mathrm{L} \text {, } \\
\text { SP: } 5.4 \mathrm{mmol} / \mathrm{L}) \\
\text { Between the two groups, } \\
\text { there were no significant } \\
\text { differences in changes of: } \\
\text { SHBG } \\
\text { Total and free } \\
\text { testosterone } \\
\text { C-peptide }\end{array}$ \\
\hline Toscani et $\mathrm{al}^{88}$ & $\begin{array}{l}22.72 \pm 5.68 \\
29.35 \pm 5.74\end{array}$ & $\begin{array}{l}8 \text { weeks } \\
\text { RCT }\end{array}$ & 40 & $\begin{array}{l}\text { HP diet } \\
\text { NP diet }\end{array}$ & $\begin{array}{l}30 \% \text { PRO } \\
40 \% \text { CHO } \\
30 \% \text { FAT } \\
20-30 \mathrm{kcal} \times \text { BW/day } \\
\text { I5\% PRO } \\
55 \% \text { CHO } \\
30 \% \text { FAT } \\
20-30 \text { kcal } \times \text { BW/ } \\
\text { day }\end{array}$ & $\begin{array}{l}\text { Decreased significantly } \\
\text { after both diets in both } \\
\text { groups: } \\
\text { BW } \\
\text { BMI } \\
\text { WC } \\
\text { Body fat \% } \\
\text { Sum of trunk skinfolds } \\
\text { No changes in lipid profile } \\
\text { with either diet. Total } \\
\text { testosterone decreased in } \\
\text { both PCOS and controls, } \\
\text { independently of diet } \\
\text { composition }\end{array}$ \\
\hline $\begin{array}{l}\text { Kasim-Karakas } \\
\text { et al }{ }^{89}\end{array}$ & $\begin{array}{l}18-45 \\
38.9 \pm 1.6 / 35.4 \pm 1.8\end{array}$ & $\begin{array}{l}2 \text { months } \\
\text { RCT }\end{array}$ & 24 & $\begin{array}{l}\text { PRO: } \\
\text { deficit of } \\
450 \mathrm{kcal} / \mathrm{day} \\
\text { (energy intake } \\
\text { was reduced } \\
\text { by } 700 \mathrm{kcal} \text { ) } \\
\text { plus a } 240-\mathrm{kcal} \\
\text { supplement } \\
\text { containing } \\
\text { whey PRO } \\
\text { Simple sugar: } \\
\text { deficit of } \\
450 \mathrm{kcal} / \mathrm{day} \\
\text { (energy intake } \\
\text { was reduced } \\
\text { by } 700 \mathrm{kcal} \text { ) } \\
\text { plus a } 240-\mathrm{kcal} \\
\text { supplement } \\
\text { containing } \\
\text { simple sugars }\end{array}$ & $\begin{array}{l}\text { I } 352 \mathrm{kcal} / \text { day } \\
16.6 \% \text { PRO } \\
56.7 \% \text { CHO } \\
25.9 \% \text { FAT }\end{array}$ & $\begin{array}{l}\text { The PRO group lost more: } \\
\text { BW }(-3.3 \pm 0.8 \mathrm{~kg} \text { vs } \\
-I . I \pm 0.6 \mathrm{~kg}) \\
\text { Fat mass }(-3 . \mathrm{I} \pm 0.9 \mathrm{~kg} \text { vs } \\
-0.5 \pm 0.6 \mathrm{~kg}) \\
\text { The PRO group had } \\
\text { greater decreases in: } \\
\text { Serum cholesterol } \\
(-33.0 \pm 8.4 \mathrm{mg} / \mathrm{dL} \text { vs } \\
-2.3 \pm 6.8 \mathrm{mg} / \mathrm{dL}) \\
\mathrm{HDL}(-4.5 \pm \mathrm{I} .3 \mathrm{mg} / \mathrm{dL} \text { vs } \\
-0.4 \pm \mathrm{I} .3 \mathrm{mg} / \mathrm{dL}) \\
\text { Apolipoprotein B } \\
(-20 \pm 5 \mathrm{mg} / \mathrm{dL} \text { vs } \\
3 \pm 5 \mathrm{mg} / \mathrm{dL}) \\
\text { There were no significant } \\
\text { changes between the two } \\
\text { groups in fasting glucose, } \\
\text { insulin, HOMA, HbAlc, } \\
\text { TGs and hs-CRP }\end{array}$ \\
\hline
\end{tabular}


Table I (Continued)

\begin{tabular}{|c|c|c|c|c|c|c|}
\hline Study & $\begin{array}{l}\text { Age (years) } \\
\text { BW category } \\
\left(\mathbf{k g} / \mathbf{m}^{2}\right)\end{array}$ & $\begin{array}{l}\text { Duration } \\
\text { and design } \\
\text { of dietary } \\
\text { intervention }\end{array}$ & $\begin{array}{l}\text { Sample } \\
\text { size }\end{array}$ & $\begin{array}{l}\text { Description } \\
\text { of groups }\end{array}$ & $\begin{array}{l}\text { Dietary } \\
\text { intervention }\end{array}$ & $\begin{array}{l}\text { Clinical outcome } \\
\text { measures }\end{array}$ \\
\hline $\begin{array}{l}\text { Mavropoulos } \\
\text { et a }{ }^{85}\end{array}$ & $\begin{array}{l}34.5 \\
38.5\end{array}$ & $\begin{array}{l}6 \text { months } \\
\text { Nonran- } \\
\text { domized } \\
\text { clinical trial } \\
\text { without a } \\
\text { control group }\end{array}$ & 5 & $\begin{array}{l}\text { LCKD } \\
(\leq 20 \mathrm{~g} \\
\mathrm{CHO} / \text { day })\end{array}$ & $\begin{array}{l}\text { Diet included } \\
\text { unlimited } \\
\text { consumption of } \\
\text { animal foods (meat, } \\
\text { chicken, turkey, other } \\
\text { fowl, fish, shellfish), } \\
\text { cheeses (up to } 4 \text { and } \\
2 \text { ounces per day) } \\
\text { unlimited eggs, non- } \\
\text { starchy vegetables } \\
\text { (two cups per day), } \\
\text { and limited intake of } \\
\text { starchy vegetables } \\
\text { (one cup per day) }\end{array}$ & $\begin{array}{l}\text { Significant reductions from } \\
\text { baseline to } 24 \text { weeks with } \\
\text { the LCKD in: } \\
\text { BW }(-12 \%) \\
\text { Percent-free testosterone } \\
(-22 \%) \\
\text { LH/FSH ratio }(-36 \%) \\
\text { Fasting insulin }(-54 \%)\end{array}$ \\
\hline Stamets et a ${ }^{142}$ & $\begin{array}{l}29 \pm 4 / 26 \pm 4 \\
38 \pm 4 / 37 \pm 5\end{array}$ & $\begin{array}{l}\text { I month } \\
\text { RCT }\end{array}$ & 26 & $\begin{array}{l}\text { HP energy- } \\
\text { restricted diet } \\
\text { (1000-kcal } \\
\text { deficit per day) } \\
\text { High- } \\
\text { carbohydrate } \\
\text { energy- } \\
\text { restricted diet } \\
\text { (1000-kcal } \\
\text { deficit per } \\
\text { day) }\end{array}$ & $\begin{array}{l}30 \% \text { PRO } \\
40 \% \text { CHO } \\
30 \% \text { FAT } \\
15 \% \text { PRO } \\
55 \% \text { CHO } \\
30 \% \text { FAT }\end{array}$ &  \\
\hline Moran et $\mathrm{al}^{41}$ & $\begin{array}{l}33 \pm 0.84 \\
37.4 \pm 1.24\end{array}$ & $\begin{array}{l}\text { I } 6 \text { weeks } \\
\text { (12 weeks } \\
\text { of energy } \\
\text { restriction, } \\
\text { followed } \\
\text { by } 4 \text { weeks } \\
\text { of weight } \\
\text { maintenance) } \\
\text { RCT }\end{array}$ & 28 & $\begin{array}{l}\text { LP: } \\
\text { I5\% PRO } \\
55 \% \text { CHO } \\
30 \% \text { FAT } \\
\text { HP: } \\
30 \% \text { PRO } \\
40 \% \text { CHO } \\
30 \% \text { FAT }\end{array}$ & $\begin{array}{l}\text { Isocaloric diets } \\
\text { energy-restricted } \\
\text { diet ( } 6000 \mathrm{~kJ} / \text { day) } \\
\text { was prescribed } \\
\text { for } 12 \text { weeks, } \\
\text { followed by a weight } \\
\text { maintenance diet } \\
\text { for the final } 4 \text { weeks } \\
\text { with the same } \\
\text { dietary composition } \\
\text { in both phases plus } \\
\text { advice for weekly } \\
\text { exercise }\end{array}$ & $\begin{array}{l}\text { Both diets resulted in } \\
\text { decreased: } \\
\text { BW }(-7.7 \pm 0.7 \mathrm{~kg}) \\
\text { Total fat mass }(-14.4 \%) \\
\text { Total lean mass }(-3.4 \%) \\
\text { Abdominal fat }(-12.5 \%) \\
\text { Total cholesterol }(-8.8 \%) \\
\text { TGs }(-12.5 \%) \\
\text { LDL-C }(-9.8 \%) \\
\text { Fasting insulin }(-20 \%) \\
\text { HOMA }(-9 \%) \\
\text { The LP diet decreased } \\
\text { HDL-C }(-10 \%) \\
\text { No association between } \\
\text { dietary composition and } \\
\text { reproductive clinical } \\
\text { parameters }\end{array}$ \\
\hline
\end{tabular}


Table I (Continued)

\begin{tabular}{|c|c|c|c|c|c|c|}
\hline Study & $\begin{array}{l}\text { Age (years) } \\
\text { BW category } \\
\left(\mathrm{kg} / \mathrm{m}^{2}\right)\end{array}$ & $\begin{array}{l}\text { Duration } \\
\text { and design } \\
\text { of dietary } \\
\text { intervention }\end{array}$ & $\begin{array}{l}\text { Sample } \\
\text { size }\end{array}$ & $\begin{array}{l}\text { Description } \\
\text { of groups }\end{array}$ & $\begin{array}{l}\text { Dietary } \\
\text { intervention }\end{array}$ & $\begin{array}{l}\text { Clinical outcome } \\
\text { measures }\end{array}$ \\
\hline Phy et $\mathrm{al}^{96}$ & $\begin{array}{l}29.8 \pm 4.0 \\
38.3 \pm 5.5\end{array}$ & $\begin{array}{l}2 \text { months } \\
\text { Nonran- } \\
\text { domized } \\
\text { clinical trial } \\
\text { without a } \\
\text { control group }\end{array}$ & 24 & $\begin{array}{l}\text { Ad libitum low } \\
\text { starch/low } \\
\text { dairy diet }\end{array}$ & $\begin{array}{l}\text { Participants were } \\
\text { instructed to eat } \\
\text { animal PRO (meat } \\
\text { and poultry), fish and } \\
\text { shellfish, eggs, non- } \\
\text { starchy vegetables, } \\
\text { low-sugar fruits } \\
\text { (berries, apples, } \\
\text { oranges, plums, etc), } \\
\text { avocados, olives, } \\
\text { nuts and seeds } \\
\text { and oils (olive and } \\
\text { coconut) }\end{array}$ & 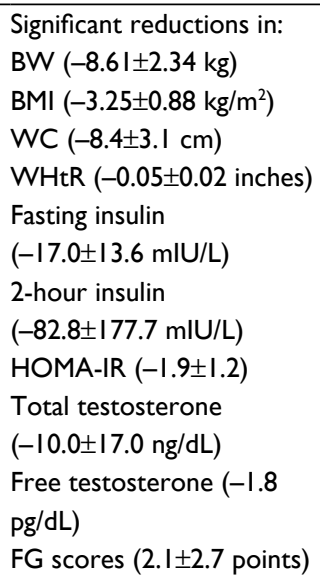 \\
\hline $\begin{array}{l}\text { Foroozanfard } \\
\text { et al }{ }^{105}\end{array}$ & $\begin{array}{l}27.1 \pm 4.7 \\
32.3 \pm 4.6\end{array}$ & $\begin{array}{l}3 \text { months } \\
\text { RCT }\end{array}$ & 53 & $\begin{array}{l}\text { Low-calorie } \\
\text { DASH diet }\end{array}$ & $\begin{array}{l}\text { Calorie-restricted } \\
\text { (350-700 kcal less) } \\
\text { I6-I8\% PRO } \\
52-55 \% \text { CHO } \\
30 \% \text { FAT } \\
\text { (rich in fruits, } \\
\text { vegetables, whole } \\
\text { grains, LF dairy } \\
\text { products and low } \\
\text { in saturated fats, } \\
\text { cholesterol, refined } \\
\text { grains and sweets) } \\
\text { Calorie-restricted } \\
\text { (350-700 kcal less) } \\
52-55 \% \text { CHO } \\
\text { I6-I8\% PRO } \\
30 \% \text { FAT } \\
\text { (different in food } \\
\text { groups contained) }\end{array}$ & 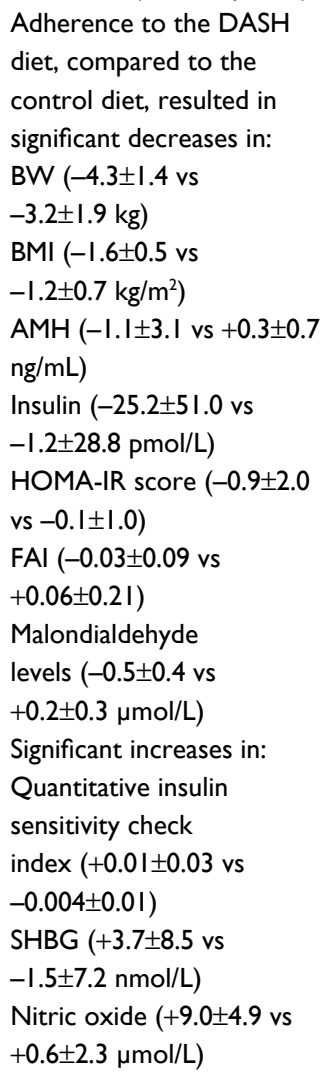 \\
\hline Perelman et $\mathrm{al}^{90}$ & $\begin{array}{l}30 \pm 7 \\
39 \pm 7\end{array}$ & $\begin{array}{l}\text { Two phases } \\
\text { of 3-week } \\
\text { isocaloric } \\
\text { program } \\
\text { RCT crossover } \\
\text { design }\end{array}$ & 6 & $\begin{array}{l}\text { Eucaloric diet } \\
\uparrow \text { poly- and } \\
\text { monounsa- } \\
\text { turated fat }\end{array}$ & $\begin{array}{l}\text { I5\% PRO } \\
40 \% \text { CHO } \\
45 \% \text { FAT } \\
7 \% \text { saturated fat } \\
\text { Ratio of poly to } \\
\text { mono=I.0 } \\
200 \mathrm{mg} \text { Cholesterol } \\
20 \text { g fiber } \\
\text { No weight loss }\end{array}$ & $\begin{array}{l}\text { AUC for insulin } \\
\text { concentrations were } \\
30 \% \text { lower on the low- } \\
\mathrm{CHO} / \text { fat-enriched diet } \\
(-194 \pm 148 \mathrm{mIU} / \mathrm{L} \times 8 \mathrm{~h}) \\
\mathrm{LDL}-\mathrm{C} \text { was significantly } \\
\text { lower on the low- } \\
\mathrm{CHO} / \text { fat-enriched diet } \\
(-12 \pm 60 \mathrm{mg} / \mathrm{dL})\end{array}$ \\
\hline
\end{tabular}


Table I (Continued)

\begin{tabular}{|c|c|c|c|c|c|c|}
\hline Study & $\begin{array}{l}\text { Age (years) } \\
\text { BW category } \\
\left(\mathrm{kg} / \mathrm{m}^{2}\right)\end{array}$ & $\begin{array}{l}\text { Duration } \\
\text { and design } \\
\text { of dietary } \\
\text { intervention }\end{array}$ & $\begin{array}{l}\text { Sample } \\
\text { size }\end{array}$ & $\begin{array}{l}\text { Description } \\
\text { of groups }\end{array}$ & $\begin{array}{l}\text { Dietary } \\
\text { intervention }\end{array}$ & $\begin{array}{l}\text { Clinical outcome } \\
\text { measures }\end{array}$ \\
\hline & & & & $\begin{array}{l}\text { Eucaloric diet } \\
\uparrow \mathrm{CHO}\end{array}$ & $\begin{array}{l}\text { I5\%PRO } \\
60 \% \text { CHO } \\
25 \% \text { FAT } \\
7 \% \text { saturated fat } \\
\text { Ratio of poly to } \\
\text { mono=I.0 } \\
200 \mathrm{mg} \text { Cholesterol } \\
20 \mathrm{~g} \text { fiber } \\
\text { No weight loss }\end{array}$ & $\begin{array}{l}\text { Fasting plasma total } \\
\text { cholesterol, TG and } \\
\text { HDL-C concentrations did } \\
\text { not differ between diets }\end{array}$ \\
\hline $\begin{array}{l}\text { Asemi and } \\
\text { Esmaillzadeh }^{78}\end{array}$ & $\begin{array}{l}30.7 \pm 6.7 / 29.4 \pm 6.2 \\
29.1 \pm 3.2 / 31.5 \pm 5.7\end{array}$ & $\begin{array}{l}2 \text { months } \\
\text { RCT }\end{array}$ & 48 & $\begin{array}{l}\text { DASH diet: } \\
\text { calorie- } \\
\text { restricted diet }\end{array}$ & $\begin{array}{l}\text { Deficit of } \\
350-700 \mathrm{kcal} / \text { day } \\
\text { I8\% PRO } \\
52 \% \text { CHO } \\
30 \% \text { FAT } \\
\text { Designed based on } \\
\text { Iranian traditional } \\
\text { dietary pattern } \\
\text { Deficit of } \\
350-700 \mathrm{kcal} / \text { day } \\
\text { I8\% PRO } \\
52 \% \text { CHO } \\
30 \% \text { FAT } \\
\text { Rich in: fruits/ } \\
\text { vegetables/whole } \\
\text { grains/LF dairy } \\
\text { products low in: } \\
\text { saturated fats/ } \\
\text { cholesterol/refined } \\
\text { grains/sweets }\end{array}$ & $\begin{array}{l}\text { Adherence to the DASH } \\
\text { eating pattern, compared } \\
\text { to the control diet, } \\
\text { resulted in significant } \\
\text { reductions in: } \\
\text { Insulin }(-1.88 \mu \mathrm{IU} / \mathrm{mL}) \\
\text { HOMA-IR }(-0.45) \\
\text { Serum hs-CRP levels } \\
(-763.29) \\
\text { WC }(-5.2 \mathrm{~cm}) \\
\text { HC }(-5.9 \mathrm{~cm})\end{array}$ \\
\hline Asemi et al ${ }^{106}$ & $\begin{array}{l}30.7 \pm 6.7 / 29.4 \pm 6.2 \\
29.1 \pm 3.2 / 31.5 \pm 5.7\end{array}$ & $\begin{array}{l}2 \text { months } \\
\text { RCT }\end{array}$ & 48 & $\begin{array}{l}\text { Control diet: } \\
\text { calorie- } \\
\text { restricted diet } \\
\\
\text { DASH diet: } \\
\text { calorie- } \\
\text { restricted diet }\end{array}$ & $\begin{array}{l}\text { Deficit of } \\
350-700 \text { kcal/day } \\
\text { I8\% PRO } \\
52 \% \text { CHO } \\
30 \% \text { FAT } \\
\text { Designed based } \\
\text { on Iranian traditional } \\
\text { dietary pattern } \\
\text { Deficit of } \\
350-700 \text { kcal/day } \\
18 \% \text { PRO } \\
52 \% \text { CHO } \\
30 \% \text { FAT } \\
\text { Rich in: fruits/ } \\
\text { vegetables/whole } \\
\text { grains/LF dairy } \\
\text { products } \\
\text { low in: saturated } \\
\text { fats/cholesterol/ } \\
\text { refined grains/sweets }\end{array}$ & $\begin{array}{l}\text { Adherence to DASH } \\
\text { diet, compared to the } \\
\text { control diet, resulted in a } \\
\text { significant decrease in: } \\
\text { BW }(-4.4 \mathrm{~kg}) \\
\text { BMI }\left(-1.7 \mathrm{~kg} / \mathrm{m}^{2}\right) \\
\text { Serum TGs }(-10.0 \mathrm{mg} / \mathrm{dL}) \\
\text { VLDL-C levels }(-2.0 \mathrm{mg} / \\
\mathrm{dL}) \\
\text { Significantly increased } \\
\text { concentrations of: } \\
\text { Plasma total antioxidant } \\
\text { capacity }(+98.6 \mathrm{mmol} / \mathrm{L}) \\
\text { Total glutathione }(+66.4 \\
\mu \text { mol/L) }\end{array}$ \\
\hline
\end{tabular}


Table I (Continued)

\begin{tabular}{|c|c|c|c|c|c|c|}
\hline Study & $\begin{array}{l}\text { Age (years) } \\
\text { BW category } \\
\left(\mathrm{kg} / \mathrm{m}^{2}\right)\end{array}$ & $\begin{array}{l}\text { Duration } \\
\text { and design } \\
\text { of dietary } \\
\text { intervention }\end{array}$ & $\begin{array}{l}\text { Sample } \\
\text { size }\end{array}$ & $\begin{array}{l}\text { Description } \\
\text { of groups }\end{array}$ & $\begin{array}{l}\text { Dietary } \\
\text { intervention }\end{array}$ & $\begin{array}{l}\text { Clinical outcome } \\
\text { measures }\end{array}$ \\
\hline Goss et a ${ }^{76}$ & $\begin{array}{l}3 I \pm 5.8 \\
31.8 \pm 5.7\end{array}$ & $\begin{array}{l}2 \text { months/ } \\
2 \text { months } \\
\text { RCT crossover } \\
\text { design }\end{array}$ & $23 / 27$ & $\begin{array}{l}\text { I800 kcal } \\
\text { Standard diet } \\
\text { I800 kcal } \\
\text { Reduced } \\
\text { CHO diet }\end{array}$ & $\begin{array}{l}\text { I8\% PRO } \\
55 \% \text { CHO } \\
27 \% \text { FAT } \\
19 \% \text { PRO } \\
41 \% \text { CHO } \\
40 \% \text { FAT }\end{array}$ & $\begin{array}{l}\text { Significant reduction in } \\
\text { body fat (reduced } \mathrm{CHO} \\
\text { diet: }-3.7 \% \text {, standard diet: } \\
-2.2 \%) \text {. } \\
\text { The reduced } \mathrm{CHO} \text { diet } \\
\text { induced a greater decrease } \\
\text { in: } \\
\text { Intra-abdominal adipose } \\
\text { tissue }(-7.1 \%) \\
\text { Subcutaneous abdominal } \\
\text { adipose tissue }(-4.6 \%) \\
\text { Intermuscular adipose } \\
\text { tissue (-1I.5\%) } \\
\text { The standard diet induced } \\
\text { a decrease in total lean } \\
\text { mass }(-1.3 \%)\end{array}$ \\
\hline Gower et al ${ }^{91}$ & $\begin{array}{l}31.2 \pm 5.8 \\
31.8 \pm 5.7\end{array}$ & $\begin{array}{l}\text { Two phases } \\
\text { of 8-week } \\
\text { isocaloric } \\
\text { program } \\
\text { RCT crossover } \\
\text { design }\end{array}$ & 27 & $\begin{array}{l}\text { Standard } \\
\text { eucaloric diet } \\
\text { Low- } \\
\text { carbohydrate } \\
\text { eucaloric diet }\end{array}$ & $\begin{array}{l}\text { I8\% PRO } \\
55 \% \text { CHO } \\
27 \% \text { FAT } \\
19 \% \text { PRO } \\
41 \% \text { CHO } \\
40 \% \text { FAT }\end{array}$ & $\begin{array}{l}\text { Low } \mathrm{CHO} \text { diet induced } \\
\text { significant decreases in: } \\
\text { Basal beta-c response } \\
\left(-1.7 \times 10^{\%} / \mathrm{min}\right) \\
\text { Fasting insulin } \\
(-15.6 \mathrm{pmol} / \mathrm{L}) \\
\text { Fasting glucose } \\
(-0.26 \mathrm{mmol} / \mathrm{L}) \\
\text { HOMA }(-0.7) \\
\text { Total testosterone } \\
(-0.55 \mathrm{nM}) \\
\text { Cholesterol } \\
(-0.53 \mathrm{mmol} / \mathrm{L}) \\
\text { LDL-C }(-0.4 \mathrm{I} \mathrm{mmol} / \mathrm{L}) \\
\text { HDL-C (-0.II mmol/L) } \\
\text { Significant increases in: } \\
\text { Insulin sensitivity index } \\
(+1.2) \\
\text { Dynamic beta-cell } \\
\text { response to glucose }(+96.1 \\
\text { I0\%/min) }\end{array}$ \\
\hline $\begin{array}{l}\text { Turner-McGrievy } \\
\text { et a }{ }^{152}\end{array}$ & $\begin{array}{l}27.8 \pm 4.5 \\
39.9 \pm 6.1\end{array}$ & $\begin{array}{l}6 \text { months } \\
\text { RCT } \\
\text { (randomized } \\
\text { controlled } \\
\text { feasibility } \\
\text { study) }\end{array}$ & 18 & $\begin{array}{l}\text { LF and LGI } \\
\text { vegan diet } \\
\text { Low-calorie } \\
\text { diet }\end{array}$ & $\begin{array}{l}\text { Plant-based diet } \\
\text { (fruits, vegetables, } \\
\text { whole grains and } \\
\text { legumes/beans, } \\
\text { excluding all animal } \\
\text { products) } \\
1200 \mathrm{kcal} / \text { day } \\
\text { for participants } \\
\text { weighing } \leq 90 \mathrm{~kg} \text { and } \\
1500 \mathrm{kcal} / \text { day for } \\
\text { participants weighing } \\
\geq 90 \mathrm{~kg}\end{array}$ & $\begin{array}{l}\text { LF, LGI vegan diet } \\
\text { produced significantly } \\
\text { greater BW losses at } \\
3 \text { months (-I.8 } \mathrm{kg} \text { ) } \\
\text { Greater improvements in } \\
\text { dietary intake at } 6 \text { months } \\
\text { (-265 kcal/day) compared } \\
\text { to a standard low-calorie } \\
\text { dietary approach } \\
\text { No significant differences } \\
\text { in changes in any of the } \\
\text { domains of the PCOS } \\
\text { Health-Related Quality } \\
\text { of Life Questionnaire } \\
\text { (emotional health, body } \\
\text { hair, BW, infertility, } \\
\text { menstrual concerns) }\end{array}$ \\
\hline
\end{tabular}


Table I (Continued)

\begin{tabular}{|c|c|c|c|c|c|c|}
\hline$\overline{\text { Study }}$ & $\begin{array}{l}\text { Age (years) } \\
\text { BW category } \\
\left(\mathrm{kg} / \mathrm{m}^{2}\right)\end{array}$ & $\begin{array}{l}\text { Duration } \\
\text { and design } \\
\text { of dietary } \\
\text { intervention }\end{array}$ & $\begin{array}{l}\text { Sample } \\
\text { size }\end{array}$ & $\begin{array}{l}\text { Description } \\
\text { of groups }\end{array}$ & $\begin{array}{l}\text { Dietary } \\
\text { intervention }\end{array}$ & $\begin{array}{l}\text { Clinical outcome } \\
\text { measures }\end{array}$ \\
\hline Douglas et $\mathrm{a}^{92}$ & $\begin{array}{l}33 \pm 6 \\
30.0 \pm 3.7\end{array}$ & $\begin{array}{l}3 \times 16 \text {-day } \\
\text { dietary } \\
\text { treatment } \\
\text { RCT crossover } \\
\text { design }\end{array}$ & 11 & $\begin{array}{l}\text { Standard } \\
\text { eucaloric diet } \\
\text { Low CHO } \\
\text { eucaloric diet } \\
\text { High MUFA } \\
\text { eucaloric diet }\end{array}$ & $\begin{array}{l}\text { I6\% PRO } \\
56 \% \text { CHO } \\
31 \% \text { FAT } \\
15 \% \text { PRO } \\
43 \% \text { CHO } \\
45 \% \text { FAT } \\
55 \% \text { CHO } \\
\text { I5\% PRO } \\
33 \% \text { FAT }\end{array}$ & $\begin{array}{l}\text { Fasting insulin was lower } \\
\text { following the Low CHO } \\
\text { diet relative to the STD } \\
\text { diet (-3.2 } \mathrm{mlU} / \mathrm{L}) \\
\text { Acute insulin response } \\
\text { to glucose was lower } \\
\text { following the low CHO } \\
\text { diet relative to the MUFA } \\
\text { diet (-97.7 mIU/L } \times \\
\text { I0 minutes) } \\
\text { No differences in: } \\
\text { Fasting glucose } \\
\text { Insulin sensitivity } \\
\text { Circulating concentrations } \\
\text { of reproductive hormones }\end{array}$ \\
\hline Moran et $\mathrm{a}^{81}$ & $\begin{array}{l}32.1 \pm 5.2 \\
34.9 \pm 7.0\end{array}$ & $\begin{array}{l}8 \text { weeks weight } \\
\text { loss } \\
6 \text { months } \\
\text { weight } \\
\text { maintenance } \\
\text { RCT } \\
\text { (Phase I: } \\
\text { all subjects } \\
\text { followed } \\
\text { the same } \\
\text { intervention, } \\
\text { Phase II: } \\
\text { randomly } \\
\text { assigned to a } \\
\text { carbohydrate } \\
\text { counting } \\
\text { group [CC], } \\
\text { with } \leq 120 \mathrm{~g} \\
\text { carbohydrate/ } \\
\text { day; or a fat } \\
\text { counting } \\
\text { [FC] group } \\
\text { with } \leq 50 \mathrm{~g} \\
\text { carbohydrate/ } \\
\text { day) }\end{array}$ & $\begin{array}{l}34 \\
\text { completed } \\
\text { Phase I } \\
23 \\
\text { completed } \\
\text { Phase II }\end{array}$ & 更 & $\begin{array}{l}8 \text { weeks of energy } \\
\text { restriction in } \\
\text { which two meals/ } \\
\text { day were replaced } \\
\text { with commercially } \\
\text { available meal } \\
\text { replacements (two- } \\
\text { meal replacements/ } \\
\text { day, } 4904.4 \pm 127 \mathrm{~kJ} \text { ) } \\
8000 \text { steps/day } \\
24 \text { weeks of } \\
\text { weight loss } \\
\text { maintenance during } \\
\text { which subjects } \\
\text { followed either } \\
\text { a carbohydrate } \\
\text { counting ( } \leq 120 \mathrm{~g} / \\
\text { day; CC) or fat } \\
\text { counting ( } \leq 50 \mathrm{~g} / \text { day; } \\
\text { FC) protocol } \\
8000 \text { steps/day }\end{array}$ & $\begin{array}{l}\text { Phase I: significant } \\
\text { reductions in } \\
\text { BW }(-5.6 \pm 2.4 \mathrm{~kg}) \\
\text { WC }(-6.1 \pm 2.5 \mathrm{~cm}) \\
\text { Body fat }(-4 . I \pm 2.2 \mathrm{~kg}) \\
\text { Insulin }(-2.8 \pm I . I \mathrm{mIU} / \mathrm{L}) \\
\text { Total testosterone } \\
(0.3 \pm 0.7 \mathrm{nmol} / \mathrm{L}) \\
\text { FAI }(-3.1 \pm 4.6) \\
\text { These changes were } \\
\text { sustained during Phase II. } \\
\text { Improvements in menstrual } \\
\text { cyclicity occurred for I6 } \\
(57.1 \%) \text { of } 28 \text { subjects. } \\
\text { Moderate fat or } \\
\text { carbohydrate restriction } \\
\text { was equally effective } \\
\text { in maintaining weight } \\
\text { reduction and improving } \\
\text { reproductive and metabolic } \\
\text { variables }\end{array}$ \\
\hline \multicolumn{7}{|c|}{ Alternative dietary interventions } \\
\hline $\begin{array}{l}\text { Papakonstantinou } \\
\text { et al }{ }^{132}\end{array}$ & $\begin{array}{l}27 \pm 6 \\
\text { Whole sample }(n=40, \\
27 \pm 6) \\
\text { Normal weight }(n=20, \\
24 \pm 1.22) \\
\text { Overweight/obese } \\
(n=20,30 \pm 1.21)\end{array}$ & $\begin{array}{l}\text { 24-week } \\
\text { crossover } \\
\text { design, RCT } \\
\text { trial }\end{array}$ & 40 & $\begin{array}{l}\text { Weight } \\
\text { maintenance, } \\
\text { isocaloric diet } \\
\text { consumed as } \\
\text { a three-meal } \\
\text { pattern } \\
\text { Weight } \\
\text { maintenance, } \\
\text { isocaloric diet } \\
\text { consumed } \\
\text { as a six-meal } \\
\text { pattern }\end{array}$ & $\begin{array}{l}25 \% \text { PRO } \\
40 \% \text { CHO } \\
35 \% \text { FAT } \\
\\
25 \% \text { PRO } \\
40 \% \text { CHO } \\
35 \% \text { FAT }\end{array}$ & $\begin{array}{l}\text { Six meals decreased } \\
\text { significantly vs three meals: } \\
\text { Fasting insulin (three } \\
\text { meals: I I } 2 \pm 10 \text { pmol/L, six } \\
\text { meals: } 92 \pm 10 \text { pmol/L) } \\
\text { Post-OGTT insulin } \\
\text { sensitivity (Matsuda index, } \\
\text { three meals: } 3.80 \pm 0.3 \mathrm{I} \text {, six } \\
\text { meals: } 5.25 \pm 0.67 \text { ) } \\
\text { Trend toward significance } \\
(p=0.063 \text { ): } \\
\text { HOMA-IR (three meals: } \\
2.94 \pm 0.25 \text {, six meals: } \\
2.45 \pm 0.3 \mathrm{I} \text { ) }\end{array}$ \\
\hline
\end{tabular}

(Continued) 
Table I (Continued)

\begin{tabular}{|c|c|c|c|c|c|c|}
\hline Study & $\begin{array}{l}\text { Age (years) } \\
\text { BW category } \\
\left(\mathrm{kg} / \mathrm{m}^{2}\right)\end{array}$ & $\begin{array}{l}\text { Duration } \\
\text { and design } \\
\text { of dietary } \\
\text { intervention }\end{array}$ & $\begin{array}{l}\text { Sample } \\
\text { size }\end{array}$ & $\begin{array}{l}\text { Description } \\
\text { of groups }\end{array}$ & $\begin{array}{l}\text { Dietary } \\
\text { intervention }\end{array}$ & $\begin{array}{l}\text { Clinical outcome } \\
\text { measures }\end{array}$ \\
\hline Jakubowicz & $25-39$ & 3 months & 51 & Isocaloric diet & $1800 \pm 25 \mathrm{kcal} / \mathrm{day}$ & The BF group has \\
\hline \multirow[t]{21}{*}{ et $\mathrm{al}^{133}$} & $23.7 \pm 0.2$ & $\mathrm{RCT}$ & & High-calorie & (42\% PRO & significant changes in: \\
\hline & & & & $\mathrm{BF}$ & $27 \% \mathrm{CHO}$ & Fasting glucose $(-8 \%)$ \\
\hline & & & & & $31 \%$ FAT) & Insulin $(-53 \%)$ \\
\hline & & & & & BF $(980 \mathrm{kcal}, 54 \%$ & HOMA-IR $(-56 \%)$ \\
\hline & & & & & daily energy intake) & HOMA-B (-35\%) \\
\hline & & & & & Lunch $(640 \mathrm{kcal}, 35 \%$ & ISI (I35\%) \\
\hline & & & & & daily energy intake) & Compared to baseline, the \\
\hline & & & & & Dinner (190 kcal, & BF group had significantly \\
\hline & & & & & II\% daily energy & lower: \\
\hline & & & & & intake) & AUC glucose $(-20 \%)$ \\
\hline & & & & Isocaloric diet & BF (I $90 \mathrm{kcal}, \mathrm{I}$ I\% & AUC insulin (-49\%) \\
\hline & & & & High-calorie & daily energy intake) & Androstenedione (-34\%) \\
\hline & & & & dinner & Lunch $(640 \mathrm{kcal}, 35 \%$ & DHEAS $(-35 \%)$ \\
\hline & & & & & daily energy intake) & Total testosterone $(-47 \%)$ \\
\hline & & & & & Dinner $(980$ kcal, & Free testosterone (from \\
\hline & & & & & $54 \%$ daily energy & $3.4 \pm 0.2$ to $1.7 \pm 0.1 \mathrm{ng} / \mathrm{dL})$ \\
\hline & & & & & intake) & Compared to baseline, the \\
\hline & & & & & & BF group had significantly \\
\hline & & & & & & higher: \\
\hline & & & & & & SHBG (from $2 \pm 0.1$ to \\
\hline & & & & & & $4.1 \pm 0.2 \mu \mathrm{g} / \mathrm{dL})$ \\
\hline
\end{tabular}

Abbreviations: $\mathrm{AMH}$, anti-Mullerian hormone; AUC, area under the curve; BF, breakfast; BMI, body mass index; BW, body weight; CC, clomiphene citrate; CHCD, conventional hypocaloric diet; CHD, conventional healthy diet; $\mathrm{CHO}$, carbohydrates; DASH, Dietary Approaches to Stop Hypertension; DHEAS, dehydroepiandrosterone sulfate; FAl, free androgen index; FAT, dietary fat content; FG, Ferriman-Gallwey; FSH, follicle-stimulating hormone; GI, glycemic index; GL, glycemic load; HbAlc, hemoglobin AI c; HC, hip circumference; HDL-C, high-density lipoprotein cholesterol; HOMA-B, homeostatic model assessment for B-cell function; HOMA-IR, homeostatic model assessment for insulin resistance; HP, high protein; hsCRP, high-sensitivity C-reactive protein; ISI, insulin sensitivity index; LCKD, low-carbohydrate ketogenic diet; LF, low fat; LGI, low glycemic index; LGL, low glycemic load; LDL-C, low-density lipoprotein cholesterol; LH, luteinizing hormone; MHCD, modified hypocaloric diet; MUFA, monounsaturated fatty acid; NEFA, non-esterified fatty acids; NP, normal protein; OGTT, oral glucose tolerance test; PCOS, polycystic ovary syndrome; PRO, protein; RCT, randomized controlled trial; SHBG, sex hormone-binding globulin; SP, standard protein; TGs, triglycerides; VLDL-C, very low-density lipoprotein cholesterol; WC, waist circumference.

\section{Options for PCOS treatment}

Typical treatment modalities used in women with PCOS include lifestyle modification (LSM; diet, physical activity and cognitive behavior therapy) and pharmacotherapy, such as clomiphene citrate (CC), aromatase inhibitors, low-dose human menopausal gonadotropin (hMG) or FSH, insulin sensitizers, laparoscopic ovarian drilling and in vitro fertilization (IVF). ${ }^{5,47-50} \mathrm{CC}$ is the first-line therapy for ovulation induction in anovulatory infertile women with PCOS, although almost $20 \%$ of patients are unresponsive. ${ }^{47,51}$ Results from a three-arm randomized, parallel, controlled, assessor-blinded clinical trial investigating the effects of a 6-week intervention with structured exercise training and hypocaloric diet on probability of ovulation after $\mathrm{CC}$ in overweight and obese CC-resistant PCOS women showed that structured exercise training combined with hypocaloric diet was effective in increasing the response to $\mathrm{CC}$ and the ovulation rate in overweight and obese PCOS patients. ${ }^{52}$ Results from a study comparing immediate treatment with
$\mathrm{CC}$ to delayed treatment with $\mathrm{CC}$ after preconception treatment with continuous oral contraceptives, LSM (including caloric restriction, antiobesity medication, behavioral modification and exercise) or the combination of both showed benefit of improved ovulation and live birth with delayed infertility treatment with CC when preceded by LSM with weight loss compared to immediate treatment. ${ }^{53}$ Moreover, a preconception lifestyle intervention leading to $6-7 \%$ weight loss eliminated the adverse metabolic oral contraceptive effects and led to higher ovulation rates. ${ }^{54}$

Aromatase inhibitors, such as letrozole, are also used for ovulation induction. ${ }^{47} \mathrm{~A}$ recent Cochrane review showed that letrozole appeared to improve live birth and pregnancy rates in subfertile women with anovulatory PCOS, compared to CC. ${ }^{55}$ There seems to be no difference in effectiveness between letrozole and laparoscopic ovarian drilling. ${ }^{55} \mathrm{~A}$ recent meta-analysis investigating the comparative efficacies of ovulation induction treatments of 26 randomized clinical trials with 2722 participants and nine types of therapies (CC, 
Table 2 Nutrition interventions with PUFAs in women with PCOS

\begin{tabular}{|c|c|c|c|c|c|c|}
\hline Study & $\begin{array}{l}\text { Age (years)/ } \\
\text { body weight } \\
\text { category } \\
\left(\mathrm{kg} / \mathrm{m}^{2}\right)\end{array}$ & $\begin{array}{l}\text { Duration } \\
\text { and design } \\
\text { of dietary } \\
\text { intervention }\end{array}$ & $\begin{array}{l}\text { Sample } \\
\text { size }\end{array}$ & $\begin{array}{l}\text { Description of } \\
\text { groups }\end{array}$ & $\begin{array}{l}\text { Characteristics/ } \\
\text { interventions }\end{array}$ & Main conclusions \\
\hline Cussons et al $\left.\right|^{117}$ & $\begin{array}{l}32.7 \pm 7.7 \\
34.8 \pm 6.8\end{array}$ & $\begin{array}{l}8 \text { weeks } \\
\text { RCT } \\
\text { Double-blind, } \\
\text { randomized } \\
\text { crossover study } \\
\text { design }\end{array}$ & 25 & n-3 PUFA & $\begin{array}{l}4 \mathrm{~g} \mathrm{n}-3 / \text { day }(4 \times \mathrm{I} g \\
\text { capsules of } 56 \% \\
\text { DHA and } 27 \% \\
\text { EPA) }\end{array}$ & $\begin{array}{l}\text { n-3 PUFA compared to placebo } \\
\text { significantly decreased: } \\
\text { Liver fat content } \\
\text { TGs } \\
\text { Systolic blood pressure } \\
\text { Diastolic blood pressure } \\
\text { Hepatic fat in women with hepatic } \\
\text { steatosis }\end{array}$ \\
\hline Karakas et al ${ }^{109}$ & $\begin{array}{l}31.7 \pm 7.8 \\
36.3 \pm 7.8\end{array}$ & $\begin{array}{l}6 \text { weeks } \\
\text { RCT } \\
\text { Three parallel- } \\
\text { arm, randomized, } \\
\text { double-blind study } \\
\text { design }\end{array}$ & 51 & Fish oil $(n=17)$ & $\begin{array}{l}\text { Fish oil ( } 3.5 \mathrm{~g} \mathrm{n}-3 \\
\text { PUFA, six capsules/ } \\
\text { day; each capsule } \\
\text { contained } 358 \mathrm{mg} \\
\text { EPA and } 242 \mathrm{mg} \\
\text { DHA) }\end{array}$ & $\begin{array}{l}\text { Effects of fish oil and soybean oil on } \\
\text { plasma aromatic amino acids were } \\
\text { similar and differed significantly } \\
\text { from those of flaxseed oil } \\
\text { Dietary PUFA may influence insulin } \\
\text { secretion and resistance directly } \\
\text { and alter plasma aromatic amino } \\
\text { acids indirectly } \\
\text { Dietary PUFA may directly affect } \\
\text { aromatic amino acid metabolism } \\
\text { and the changes in insulin secretion } \\
\text { and resistance may be secondary }\end{array}$ \\
\hline Khani et al ${ }^{120}$ & $\begin{array}{l}31.0 \pm 5.0 \\
29.2 \pm 6.7\end{array}$ & $\begin{array}{l}6 \text { months } \\
\text { Double-blind } \\
\text { Placebo- } \\
\text { controlled }\end{array}$ & 87 & $\begin{array}{l}n-3 \text { PUFA }(n=43) \\
\text { Placebo }(n=44)\end{array}$ & $\begin{array}{l}2 \mathrm{~g} \mathrm{n}-3 \text { (two } \\
\text { capsules/day) } \\
\text { containing } 180 \mathrm{~g} \\
\text { EPA and } 120 \mathrm{mg} \\
\text { DHA } \\
\text { Two olive oil } \\
\text { capsules }\end{array}$ & $\begin{array}{l}\text { n-3 PUFA compared to placebo } \\
\text { decreased: } \\
\text { BMI } \\
\text { WC } \\
\text { TG } \\
\text { Interval between periods } \\
\text { n-3 PUFA compared to placebo } \\
\text { increased: } \\
\text { HDL }\end{array}$ \\
\hline $\begin{array}{l}\text { Kalgaonkar } \\
\text { et al }{ }^{108}\end{array}$ & $\begin{array}{l}36.2 \pm I .7 \\
35.1 \pm I .8\end{array}$ & $\begin{array}{l}6 \text { weeks } \\
\text { RCT } \\
\text { Parallel, } \\
\text { randomized } \\
\text { controlled study } \\
\text { design }\end{array}$ & 31 & Almonds & $\begin{array}{l}46 \mathrm{~g} \text { of almonds } \\
(2.4 \mathrm{~g} \text { saturated fat, } \\
19.5 \mathrm{~g} \\
\text { MUFA, } 7.5 \mathrm{~g} \\
\text { linoleic acid) }\end{array}$ & $\begin{array}{l}\text { Walnuts increased the } n-3 / n-6 \\
\text { essential PUFA in the diet and } \\
\text { plasma phospholipids } \\
\text { Walnuts significantly decreased } \\
\text { LDL-C }(-6 \%) \text { and apolipoprotein } \\
\text { B (-II\%) } \\
\text { Walnuts significantly increased } \\
\text { insulin response during OGTT } \\
(26 \%) \\
\text { Both walnuts and almonds } \\
\text { significantly increased adiponectin } \\
\text { Walnuts decreased HbAIc with } \\
\text { significant intergroup difference } \\
\text { from almonds } \\
\text { Walnuts increased SHBG and } \\
\text { almonds reduced FAI }\end{array}$ \\
\hline Nasri et al ${ }^{113}$ & $\begin{array}{l}27.5 \pm 5.7 \\
27.1 \pm 4.6\end{array}$ & $\begin{array}{l}\text { I } 2 \text { weeks } \\
\text { RCT }\end{array}$ & 60 & $n-3$ PUFA $(n=30)$ & $\begin{array}{l}2 \mathrm{~g} \mathrm{n}-3 \text { from } \\
\text { flaxseed oil } \\
\text { containing } 800 \mathrm{mg} \\
\text { ALA/day }\end{array}$ & $\begin{array}{l}\mathrm{n}-3 \text { PUFA supplementation } \\
\text { had beneficial effects on gene } \\
\text { expression of PPAR- } \gamma \text { and LDLR, } \\
\text { gene expression involved in insulin } \\
\text { and lipid signaling pathways }\end{array}$ \\
\hline
\end{tabular}


Table 2 (Continued)

\begin{tabular}{|c|c|c|c|c|c|c|}
\hline Study & $\begin{array}{l}\text { Age (years)/ } \\
\text { body weight } \\
\text { category } \\
\left(\mathrm{kg} / \mathrm{m}^{2}\right)\end{array}$ & $\begin{array}{l}\text { Duration } \\
\text { and design } \\
\text { of dietary } \\
\text { intervention }\end{array}$ & $\begin{array}{l}\text { Sample } \\
\text { size }\end{array}$ & $\begin{array}{l}\text { Description of } \\
\text { groups }\end{array}$ & $\begin{array}{l}\text { Characteristics/ } \\
\text { interventions }\end{array}$ & Main conclusions \\
\hline & & $\begin{array}{l}\text { Parallel, } \\
\text { randomized, } \\
\text { double-blind, } \\
\text { placebo- } \\
\text { controlled study } \\
\text { design }\end{array}$ & & $\begin{array}{l}\text { Placebo }(n=30) \\
\text { Flaxseed oil } \\
(n=17)\end{array}$ & $\begin{array}{l}\text { Paraffin } \\
\text { Flaxseed oil ( } 3.5 \mathrm{~g} \\
\mathrm{n}-3 \text { PUFA, six } \\
\text { capsules/day; each } \\
\text { capsule contained } \\
545 \mathrm{mg} \text { ALA) } \\
\text { Soybean oil (six } \\
\text { capsules/day; each } \\
\text { capsule contained } \\
200 \text { mg oleic acid, } 429 \\
\text { mg LA, } 57 \text { mg ALA) }\end{array}$ & \\
\hline $\begin{array}{l}\text { Nadjarzadeh } \\
\text { et al }{ }^{12}\end{array}$ & $\begin{array}{l}26.92 \pm 5.46 \\
31.69 \pm 4.84\end{array}$ & $\begin{array}{l}8 \text { weeks } \\
\text { RCT } \\
\text { Parallel, } \\
\text { randomized, } \\
\text { double-blind, } \\
\text { placebo- } \\
\text { controlled study } \\
\text { design }\end{array}$ & 78 & $\begin{array}{l}n-3(n=39) \\
\text { Placebo }(n=39)\end{array}$ & $\begin{array}{l}3 \text { g/day } n-3 \\
\text { Paraffin }\end{array}$ & $\begin{array}{l}\text { n-3 PUFAs significantly decreased } \\
\text { testosterone compared to the } \\
\text { placebo group } \\
\text { FAl and the concentration of sex } \\
\text { hormone-binding protein did not } \\
\text { differ }\end{array}$ \\
\hline Oner et al ${ }^{115}$ & $\begin{array}{l}22.6 \pm 4.75 \\
22.4 \pm 3.1\end{array}$ & $\begin{array}{l}6 \text { months } \\
\text { Nonrandomized } \\
\text { clinical trial }\end{array}$ & 45 & n-3 PUFA & 1.5 g/day n-3 & $\begin{array}{l}\text { Insulin levels and HOMA-IR } \\
\text { significantly decreased during } \\
\text { treatment } \\
\text { Serum LH, total testosterone, free } \\
\text { testosterone and androstenedione } \\
\text { levels decreased significantly } \\
\text { SHBG levels and TNF- } \alpha \text { levels were } \\
\text { significantly increased at } 6 \text { months }\end{array}$ \\
\hline $\begin{array}{l}\text { Mohammadi } \\
\text { et al }{ }^{1 / 4}\end{array}$ & $\begin{array}{l}27.3 \pm 4.27 \\
28.7 \pm 3.21\end{array}$ & $\begin{array}{l}8 \text { weeks } \\
\text { RCT } \\
\text { Parallel, double- } \\
\text { blind, randomized } \\
\text { controlled study } \\
\text { design }\end{array}$ & 61 & $\begin{array}{l}n-3(n=30) \\
\text { Placebo }(n=31)\end{array}$ & $\begin{array}{l}4 \mathrm{~g} \mathrm{n}-3 / \text { day }(720 \mathrm{mg} \\
\text { EPA and } 480 \mathrm{mg} \\
\text { DHA) } \\
\text { Paraffin }\end{array}$ & $\begin{array}{l}\text { Supplementation with n-3 PUFA } \\
\text { compared to placebo: } \\
\text { Increased adiponectin }(19.5 \%) \text { and } \\
\text { HDL-C }(7.4 \%) \\
\text { Glucose }(-11.4 \%) \\
\text { Insulin }(-8.4 \%) \\
\text { HOMA-IR }(-21.8 \%) \\
\text { TC }(-8.1 \%) \\
\text { LDL-C }(-14.9 \%)\end{array}$ \\
\hline Vargas et al $\left.\right|^{116}$ & $\begin{array}{l}31.7 \pm 7.8 \\
36.3 \pm 7.8\end{array}$ & $\begin{array}{l}6 \text { weeks } \\
\text { RCT } \\
\text { Three parallel- } \\
\text { arm, randomized, } \\
\text { double-blind, } \\
\text { placebo- } \\
\text { controlled study }\end{array}$ & 51 &  & $\begin{array}{l}\text { Fish oil ( } 3.5 \mathrm{~g} \mathrm{n}-3 \\
\text { PUFA, six capsules/ } \\
\text { day; each capsule } \\
\text { contained } 358 \mathrm{mg} \text { EPA } \\
\text { and } 242 \mathrm{mg} \text { DHA) } \\
\text { Flaxseed oil ( } 3.5 \mathrm{~g} \text { n-3 } \\
\text { PUFA, six capsules/day; } \\
\text { each capsule contained } \\
545 \mathrm{mg} \text { ALA) } \\
\text { Soybean oil (six } \\
\text { capsules/day; each } \\
\text { capsule contained } \\
200 \mathrm{mg} \text { oleic acid, } \\
429 \mathrm{mg} \text { LA, } 57 \mathrm{mg} \text { ALA) }\end{array}$ & $\begin{array}{l}\text { Fish oil and flaxseed oil lowered } \\
\text { TGs } \\
\text { Soybean oil increased glucose } \\
\text { at } 30 \text { and } 60 \text { min and AUC for } \\
\text { glucose during OGTT and reduced } \\
\text { testosterone } \\
\text { Fish oil significantly increased } \\
\text { glucose at I } 20 \text { min of OGTT and } \\
\text { significantly decreased Matsuda } \\
\text { index }\end{array}$ \\
\hline
\end{tabular}

(Continued) 
Table 2 (Continued)

\begin{tabular}{|c|c|c|c|c|c|c|}
\hline Study & $\begin{array}{l}\text { Age (years)/ } \\
\text { body weight } \\
\text { category } \\
\left(\mathrm{kg} / \mathrm{m}^{2}\right)\end{array}$ & $\begin{array}{l}\text { Duration } \\
\text { and design } \\
\text { of dietary } \\
\text { intervention }\end{array}$ & $\begin{array}{l}\text { Sample } \\
\text { size }\end{array}$ & $\begin{array}{l}\text { Description of } \\
\text { groups }\end{array}$ & $\begin{array}{l}\text { Characteristics/ } \\
\text { interventions }\end{array}$ & Main conclusions \\
\hline \multirow[t]{16}{*}{ Phelan et al ${ }^{110}$} & $18-40$ years & 6 weeks & 22 & LC n-3 PUFA & $4 \times I$ g capsules/day & LC $n-3$ PUFA supplementation \\
\hline & $35.07 \pm 6.10$ & $\mathrm{RCT}$ & & supplementation & $2.4 \mathrm{~g}$ LC $\mathrm{n}-3$ PUFAs & increased plasma concentrations \\
\hline & & Randomized & & & that contained $1.9 \mathrm{~g}$ & of n-3 PUFAs and had an \\
\hline & & double-blind, & & & EPA and DHA/d in & antiandrogenic effect in PCOS \\
\hline & & placebo- & & & a ratio of EPA to & Significant increase in plasma EPA \\
\hline & & controlled, & & & DHA of I.49:1 & and DHA concentrations after LC \\
\hline & & $\begin{array}{l}\text { crossover design } \\
\text { study }\end{array}$ & & Placebo olive oil & $\begin{array}{l}4 \times 1 \text { g olive oil } \\
\text { capsules/day }\end{array}$ & $\begin{array}{l}\text { n-3 PUFA supplementation } \\
\text { Bioavailable testosterone }\end{array}$ \\
\hline & & & & Walnuts & $36 \mathrm{~g}$ of walnuts & concentrations were significantly \\
\hline & & & & & (2.9 g saturated fat, & reduced after LC n-3 PUFA \\
\hline & & & & & $4.5 \mathrm{~g}$ MUFA, $19.2 \mathrm{~g}$ & supplementation than after placebo \\
\hline & & & & & LA and $4.3 \mathrm{~g}$ ALA) & intake \\
\hline & & & & Placebo & Olive oil & \\
\hline & & & & & $(4 \times 1000 \mathrm{mg}$ & \\
\hline & & & & & capsules of olive & \\
\hline & & & & & oil containing $67 \%$ & \\
\hline & & & & & oleic acid) & \\
\hline \multirow{10}{*}{$\begin{array}{l}\text { Kasim-Karakas } \\
\text { et al }\left.\right|^{118}\end{array}$} & $34 \pm 5$ & 3 months control & 17 & Walnuts & $48 \mathrm{~g}$ walnuts $/ 800 \mathrm{kcal}$ & PUFA-rich diet significantly \\
\hline & $34.0 \pm 1.9$ & and then 3 months & & & energy intake $(19 \mathrm{~g}$ & increased fasting glucose and AUC \\
\hline & & intervention & & & LA and $3.3 \mathrm{~g}$ ALA) & for glucose \\
\hline & & Nonrandomized & & & & Fasting plasma-free fatty acids \\
\hline & & clinical trial & & & & decreased and ketone bodies \\
\hline & & & & & & decreased \\
\hline & & & & & & Plasma testosterone, free \\
\hline & & & & & & testosterone, SHBG, DHEAS, \\
\hline & & & & & & $\mathrm{LH}, \mathrm{FSH}$, and urinary estrogen \\
\hline & & & & & & conjugates did not change \\
\hline
\end{tabular}

Abbreviations: ALA, $\alpha$-linolenic acid; AUC, area under the curve; BMI, body mass index; DHA, docosahexaenoic acid; DHEAS, dehydroepiandrosterone sulfate; EPA, eicosapentaenoic acid; FAl, free androgen index; FSH, follicle-stimulating hormone; HbAlc, hemoglobin AIc; HDL-C, high-density lipoprotein cholesterol; HOMA-IR, homeostatic model assessment for insulin resistance; LA, linoleic acid; LC, long-chain fatty acids; LDL-C, low-density lipoprotein cholesterol; LDLR, low-density lipoprotein receptor; LH, luteinizing hormone; MUFA, monounsaturated fatty acid; OGTT, oral glucose tolerance test; PCOS, polycystic ovary syndrome; PPAR- $\gamma$, peroxisome proliferator-activated receptor gamma; PUFAs, polyunsaturated fatty acids; RCT, randomized controlled trial; SHBG, sex hormone-binding globulin; TC, total cholesterol; TGs, triglycerides; TNF-a, tumor necrosis factor-alpha; WC, waist circumference.

metformin, letrozole, FSH, hMG, unilateral laparoscopic ovarian drilling, bilateral laparoscopic ovarian drilling, the combination of metformin with letrozole and the combination of metformin with $\mathrm{CC}$ ) showed that FSH, hMG and the combination of metformin with letrozole were potentially more effective therapies in improving reproductive outcomes compared to the other therapies. ${ }^{56}$ Obesity seems to affect the responsiveness to $\mathrm{hMG}$ and $\mathrm{FSH}$ with increased dose needed, although this needs further investigation. ${ }^{5,47}$

Insulin sensitizers, such as metformin, are recommended for women with PCOS with impaired glucose tolerance. ${ }^{51} \mathrm{~A}$ meta-analysis of 12 randomized controlled trials (RCTs) with 608 women with PCOS showed that the combination of lifestyle intervention and metformin was associated with lower BMI and subcutaneous adipose tissue and improved menstruation compared to lifestyle and placebo over 6 months, without significant differences between metformin alone compared to lifestyle on body weight loss. ${ }^{57}$

IVF is a third-line treatment for PCOS indicated in those who have not responded to first- or second-line ovulation induction therapies or in those who require IVF for other indications and can be used with the safer gonadotropinreleasing hormone antagonist protocol and metformin as an adjunct, although no ideal protocol has been identified yet and no dietary associations with IVF have been reported. ${ }^{5,47,58}$ Obesity increases the risk of conception failure with IVF. ${ }^{5,47,58}$ Fertility in women with PCOS is maintained until the age of 38 years using IVF, but thereafter pregnancy rates decrease. ${ }^{59}$ Women with PCOS who conceive singleton pregnancies after IVF may be at increased risk of pregnancy complications and may require close antenatal monitoring. ${ }^{7,60}$ In conclusion, PCOS therapy should be individualized. ${ }^{50,60}$ There is 
a need for well-designed, long-term, with adequate sample size, studies to evaluate the effectiveness of pharmacological PCOS treatments, alone or in combination with dietary interventions to ameliorate PCOS symptoms and fertility outcomes.

\section{LSM programs and PCOS}

Lifestyle intervention (diet plus physical activity) leading to a small to moderate weight loss of about $5-10 \%$ is sufficient to significantly improve IR, restore ovulation and improve menstrual regularity and conception, as well as ameliorate hyperandrogenism, hirsutism and dyslipidemia. ${ }^{15,16,48,49,58,60-64}$ Results from a cross-sectional study showed that women with PCOS were more likely to follow a number of both healthy body weight management practices (balanced diet, reduced fat or sugar intake, adoption of a low-GI diet) as well as paradoxical nonhealthy attitudes (ie, increased smoking, use of laxative and diet pills) compared to women without PCOS. ${ }^{65}$

Although most clinicians are in favor of lifestyle changes for the management of PCOS, the majority of women with PCOS reported that they rarely receive lifestyle advice from their therapists. ${ }^{66,67}$ LSM programs combine dietary recommendations aiming at a small-to-moderate weight loss or maintenance, physical activity patterns and behavioral and cognitive approaches. Several studies have shown that LSM programs may prevent or delay the onset of type 2 diabetes in people with impaired glucose tolerance and women with PCOS. ${ }^{15,68,69}$ LSM programs, including diet and exercise, with or without dedicated stress reduction techniques, may offer significant benefits for amelioration in anthropometric, reproductive (hyperandrogenism, menstrual function, ovulation, pregnancy, conception), metabolic (glucose metabolism, IR, dyslipidemia) and quality of life parameters. ${ }^{38,48,49}$

Results from five meta-analyses of RCTs with women with PCOS supported the benefits of LSM programs on decreased BMI, fasting blood glucose, IR, FSH, sex hormone-binding globulin (SHBG), total testosterone, androstenedione, free androgen index (FAI) and Ferriman-Gallwey (FG) score and improved cardiorespiratory fitness, but not on glucose tolerance or lipid profiles..$^{57,63,70-72}$ No studies have assessed the effects of LSM programs on clinical reproductive outcomes, quality of life or treatment satisfaction in women with PCOS. ${ }^{2,70}$ A 1-year randomized, placebo-controlled trial compared the effects of four interventions: 1) metformin $850 \mathrm{mg}$ two times daily, 2) LSM plus metformin $850 \mathrm{mg}$ two times daily, 3) LSM plus placebo and 4) placebo group on anthropometric and hormonal parameters, and showed that the combined LSM plus metformin group offered greater changes in body weight $(-9 \mathrm{~kg})$, total testosterone $(-27 \mathrm{ng} /$ dL) and FAI (-9). ${ }^{73}$ Results from a study investigating the effects of a 3-month LSM in overweight/obese women with PCOS with anovulation, hyperandrogenism and polycystic ovaries compared to BMI-matched regularly menstruating controls, normal weight PCOS and normal weight controls showed that women with PCOS exhibited lower levels of insulin receptor substrate-1 and glucose transporter-4 (GLUT4) mRNA in their proliferative endometrium compared to BMI-matched controls, whereas LSM led to decreased body weight, improved menstrual regularity and increased endometrial insulin sensitivity. ${ }^{61}$

In conclusion, LSM (diet plus regular physical activity) should be the first-line strategy for the management of PCOS. The main challenge remains the long-term maintenance of healthy dietary attitudes and insulin sensitivity. A team-based approach along with lifestyle interventions may be more suitable for the management and treatment of PCOS. ${ }^{49,58,74,75}$

\section{Negative energy balance and PCOS}

Several studies assessing the effects of caloric restriction along with modified dietary macronutrient composition on multiple health outcomes and biochemical indices have shown that there is no optimal dietary macronutrient composition or dietary pattern for PCOS. ${ }^{38,41,76}$ Overall, there is little variation in weight loss with different diets, and this variation may be due to the differences in compliance and not how the body handles different macro- or micronutrients. Negative energy balance (with a deficit of 350-1000 kcal/day) seems to be the key factor leading to successful body weight and fat loss and amelioration of menstrual cycle and insulin sensitivity, irrespectively of the adopted dietary pattern. ${ }^{42,77-82}$

Results of a 1-month trial comparing the effects of two hypocaloric diets, differing in macronutrients, on a variety of clinical measures in women with PCOS, showed that negative energy balance alone resulted in significant weight loss $(-4 \mathrm{~kg})$ and decreased testosterone ( $-9 \mathrm{ng} / \mathrm{dL})$, fasting insulin $(-5 \mathrm{mIU} / \mathrm{L})$, area under the curve (AUC) for insulin $(-5.823 \mathrm{mIU} / \mathrm{L} \cdot \mathrm{min})$, fasting leptin $(-11 \mathrm{ng} / \mathrm{mL})$, AUC for leptin $(-1.854 \mathrm{ng} / \mathrm{mL} \cdot \mathrm{min})$, total cholesterol $(-22 \mathrm{mg} / \mathrm{dL})$ and low-density lipoprotein (LDL) cholesterol $(-12 \mathrm{mg} / \mathrm{dL})$, independently of macronutrient composition of the tested diets. ${ }^{42}$ Similarly, results from another trial comparing the effects of two hypocaloric diets with different macronutrient composition and GI in women with PCOS showed that negative energy balance resulted in successful weight loss $(-4 \%)$, decreased dehydroepiandrosterone sulfate (DHEAS) and increased SHBG concentrations. ${ }^{83}$ Moreover, results from 
a 6-month study comparing the effects of two hypocaloric diets (energy deficit -500 kcal/day), differing in macronutrient composition, showed that negative energy balance per se resulted in body weight and fat loss, amelioration of menstrual dysfunction (more regular menstrual episodes reported after weight loss) and hirsutism. ${ }^{77}$

In conclusion, negative energy balance is a key strategy for the management and treatment of PCOS. The size of the caloric deficit should be determined according to the individuals' needs (dietary preferences, habits, culture and metabolic goals) and physical activity patterns.

\section{Dietary carbohydrates and PCOS}

The "low-carbohydrate diet" is typically defined as having $<20 \%$ carbohydrates (equivalent to $20-60$ g carbohydrates/ day).$^{84}$ Out of 12 studies reporting effects of low-carbohydrate diets, only one, nonrandomized 24-week trial with small sample size met the criteria of the low-carbohydrate definition with PCOS subjects instructed to limit carbohydrate intake to $\leq 20 \mathrm{~g} /$ day ${ }^{85}$ One trial included a diet with $<30 \%$ carbohydrates, six trials included diets with $40 \%$ carbohydrates and four studies with $41-45 \%$ carbohydrates. ${ }^{41,42,76,83,86-92}$

Douglas et al ${ }^{92}$ compared the effects of three eucaloric diets: 1) standard (16\% protein, $56 \%$ carbohydrate, $31 \%$ fat), 2) moderately low-carbohydrate (15\% protein, $43 \%$ carbohydrate, $45 \%$ fat) and 3 ) high monounsaturated fatty acid (MUFA) diets (15\% protein, 55\% carbohydrate, 33\% fat) on glucose and insulin responses in women with PCOS and reported greater reductions in fasting insulin $(-3 \mathrm{mIU} / \mathrm{L})$ and lower acute insulin response to glucose $(-98 \mathrm{mIU} / \mathrm{L} \times$ 10 minutes) with the moderately low-carbohydrate diet compared to other two diets. In contrast, results from two clinical trials reported no significant differences in glucose or energy metabolism with moderately low-carbohydrate diets. ${ }^{41,42}$

Carbohydrate distribution may be a significant component for glucose metabolism and IR. One study provided evidence showing that the consumption of the majority of carbohydrates $(50 \%)$ at lunch time resulted in the lowest postprandial glucose spikes and improved glycemic control, compared to the majority of carbohydrates consumed at breakfast, dinner or equally distributed throughout the day, in people with type 2 diabetes. ${ }^{93}$ Moreover, another study showed that consumption of a high-carbohydrate breakfast ( $>45 \%$ of energy from carbohydrates) may have detrimental effects in people with impaired glucose regulation and it should be avoided. ${ }^{94}$

In conclusion, there is no optimum amount of carbohydrate intake for women with PCOS, and, therefore, any range of dietary carbohydrates may be adopted, according to the individuals' dietary assessment, metabolic goals, dietary habits and preferences. However, it may be advantageous to consume the majority of carbohydrates at lunch time, with the second best option, ie, their equal distribution in meals throughout the day, and to avoid a high-carbohydrate breakfast.

\section{GI, glycemic load (GL) and PCOS}

Five available clinical trials examined the effects of low GI on glucose or energy metabolism and hormonal responses in women with PCOS and reported successful weight loss, irrespective of GI. ${ }^{77,79,83,86,95}$ Out of the five trials, two showed increased insulin sensitivity ${ }^{79,95}$ and one showed decreased insulin levels, IR, DHEAS and high-sensitivity C-reactive protein (hsCRP) with adoption of low-GI diet. ${ }^{83}$

Results from one trial showed that a high-protein, moderately low-carbohydrate with low and medium GL resulted in significant reductions in blood insulin $(-4 \mathrm{mIU} / \mathrm{L}), \mathrm{IR}$ (homeostatic model assessment for IR [HOMA-IR], -0.8) and hsCRP $(-0.9 \mathrm{mg} / \mathrm{L})$ levels compared to a conventional diet. ${ }^{83}$ Similarly, results from a 12 -month dietary intervention with two similar macronutrient composition, energyreduced, low-fat, low-saturated fat, moderate-to-high fiber diets (23\% protein, $50 \%$ carbohydrates, $27 \%$ fat), low or high GI, showed that the low-GI diet provided a threefold greater improvement in whole-body insulin sensitivity and improved menstrual regularity and better emotional scores, compared to a conventional hypocaloric, low-fat diet. ${ }^{79}$ However, both the low-GI and the conventional diets led to similar improvements or changes in blood lipids and androgenic hormone concentrations, markers of inflammation and other measures of quality of life. ${ }^{79}$ Wong et al ${ }^{86}$ showed that a low-GI diet led to a greater decrease in BMI percentile in adolescents with PCOS. In a nonrandomized clinical trial, participants replaced high-GI and medium-GI with low-GI foods, and findings showed possible improvements in metabolic risk factors (ie, insulin sensitivity). ${ }^{95}$

In conclusion, results from a few available studies suggest that consumption of low-GI foods may have a small additional beneficial impact on some outcome measures in women with PCOS.

\section{Dietary protein and PCOS}

Out of seven available trials comparing the effects of highprotein hypocaloric diets compared to low-protein diets on several outcomes in women with PCOS, four found no significant effects of dietary protein on indices of energy metabolism or androgenic parameters, ${ }^{42,80,85,96}$ two reported 
greater weight loss with higher protein intake ${ }^{87,89}$ and three reported greater reductions in body fat with higher protein diets. ${ }^{87-89}$ Only one trial reported greater reductions in glucose levels with higher protein diets. ${ }^{87}$

Some studies have attempted to separate the effects of high-protein intake (from diet alone or supplementation) from low-carbohydrate intake (diets typically with $\geq 20 \%$ protein, $\leq 45 \%$ carbohydrates, varying in fat content). Two studies found greater improvements in anthropometric and other outcome measures with high-protein diets, ${ }^{87,89}$ whereas three found no differences between high- and low-protein diets. ${ }^{41,42,88}$ Sørensen et $\mathrm{l}^{87}$ demonstrated that a high-protein, low-carbohydrate diet ( $\sim 40 \%$ protein, $30 \%$ carbohydrate, $30 \%$ fat) decreased significantly more body weight $(-4.4 \mathrm{~kg})$, fat mass $(-4.3 \mathrm{~kg})$, WC $(-3.7 \mathrm{~cm})$ and glucose concentrations $(-0.2 \mathrm{mmol} / \mathrm{L})$ compared to a low-protein, high-carbohydrate diet $(\sim 15 \%$ protein, $55 \%$ carbohydrate, $30 \%$ fat). However, no differences between diets were found for SHBG, total and free testosterone and C-peptide. ${ }^{87}$ Toscani et a ${ }^{88}$ compared the effects of two diets, a high-protein, moderately low-carbohydrate diet (30\% protein, $40 \%$ carbohydrate, $30 \%$ fat) or a low-protein, highcarbohydrate ( $15 \%$ protein, $55 \%$ carbohydrate, $30 \%$ fat) diet, and reported equal reductions in body weight, BMI, WC, body fat and the sum of trunk skinfolds with both diets. Similarly, results from another study comparing the effects of a highprotein, moderately low-carbohydrate ( $30 \%$ protein, $40 \%$ carbohydrate, $30 \%$ fat) vs a low-protein, high-carbohydrate diet ( $15 \%$ protein, $55 \%$ carbohydrate, $30 \%$ fat) showed successful weight loss and improvement in reproductive and metabolic abnormalities with both diets. ${ }^{41}$

Results from one trial comparing the effects of a high whey protein, moderately low-carbohydrate diet vs normal protein, high simple carbohydrate diet on glucose metabolism, body composition, blood lipids and indices of inflammation in patients with PCOS, using two types of hypocaloric diets, a high-protein, moderately low-carbohydrate ( $\sim 34 \%$ protein, $40 \%$ carbohydrate, $26 \%$ fat) or a low-protein, high simple carbohydrate diet $(\sim 17 \%$ protein, $57 \%$ carbohydrate, $26 \%$ fat), showed greater weight and fat mass loss with the high whey protein, moderately lowcarbohydrate diet. ${ }^{89}$ However, no differences were found for fasting glucose, insulin, IR, glycated hemoglobin A1c (HbAlc), triglycerides (TGs) or hsCRP between diets. ${ }^{89} \mathrm{~A}$ meta-analysis of RCTs suggested that high-protein, moderately low-carbohydrate diets (within 6 months) may have some beneficial effects on weight loss, HbA1c and blood pressure in patients with impaired glucose metabolism. ${ }^{97}$ Moreover, our group has shown that, in obese people with and without type 2 diabetes, dietary protein did not have an important effect on glucose metabolism, ${ }^{98}$ but others have shown that it increases insulin response. ${ }^{99}$ Some proposed mechanisms for the beneficial effects of higher protein diets on body composition and weight loss include increased protein-induced thermogenesis ${ }^{100,101}$ and increased satiety, possibly due to enhanced cholecystokinin production. ${ }^{102,103}$ Moreover, a meta-analysis of RCTs reported that the majority of RCTs (35 out of 51) demonstrated a benefit of fat-free mass preservation with higher protein diets. ${ }^{104}$

In conclusion, although some studies support that higher protein diets may result in several positive health outcomes, including lean mass preservation during weight loss and maintenance, better glycemic control and amelioration of other cardiovascular disease risk factors, such as blood pressure, it is not clear yet whether these effects are due to the higher protein or lower carbohydrate intake, and no recommendations can be made currently. Nevertheless, the addition of 7-15 $\mathrm{g}$ of dietary protein in meals and snacks may offer some additional health benefits for women with PCOS, including amelioration of insulin sensitivity and lower postprandial glucose fluctuations, but this still needs to be confirmed.

\section{Dietary fat and PCOS}

Eight clinical trials compared the effects of hypocaloric lowvs high-fat diets, typically using diets with a mean of $20-30 \%$ energy from fat or dietary patterns (ie, Dietary Approaches to Stop Hypertension [DASH]), on energy metabolism in women with PCOS, and all reported significant reductions in body weight and composition with adoption of low-fat diets. $^{76,78,90-92,105,106,152}$

Moderately low-carbohydrate, high-fat diets have been shown to decrease fasting insulin and AUC for insulin and to increase insulin sensitivity in three trials in women with PCOS..$^{90-92}$ In one trial, a high-fat, moderately low-carbohydrate eucaloric diet resulted in greater reductions in body fat, intra-abdominal adipose tissue, subcutaneous abdominal adipose tissue and intermuscular adipose tissue compared to a control diet. ${ }^{76}$ Results from another trial comparing two isocaloric diets - a low-fat, low-saturated fat, high-carbohydrate ( $\sim 15 \%$ protein, $60 \%$ carbohydrate, $25 \%$ fat, $7 \%$ saturated fat) or a high-fat, low-saturated fat, moderately low-carbohydrate diet ( $\sim 15 \%$ protein, $40 \%$ carbohydrate, $45 \%$ fat, $7 \%$ saturated fat) - showed a $30 \%$ greater reduction in AUC for insulin and blood lipids with the high-fat, low-saturated fat, moderately low-carbohydrate diet, in the absence of weight loss. ${ }^{90}$ Results from a short-term, crossover trial showed that a moderately low-carbohydrate (43\%), high unsaturated fatty acids (mono- 
unsaturated fatty acids, MUFA: $18 \%$; polyunsaturated fatty acids, PUFA: 17\%) diet, led to a greater decrease in fasting insulin concentrations and the acute insulin to glucose response compared to the control diet. ${ }^{92}$ Results from another crossover clinical trial showed that a high-fat and moderately low-carbohydrate ( $\sim 1 \%$ carbohydrate, $19 \%$ protein and $40 \%$ fat) diet induced significant glucose metabolism improvements (decreased basal $\beta$-cell response, fasting insulin, fasting glucose and IR), hormonal responses (lower testosterone) and decreased blood lipids and adipose tissue (intra-abdominal adipose tissue [-7.1\%], subcutaneous abdominal adipose tissue $[-4.6 \%]$ and intermuscular adipose tissue $[-11.5 \%]){ }^{76,91}$

DASH diet is a low-GI, low-energy-density, high in complex carbohydrates, high fiber diet ( $\sim 18 \%$ protein, $52 \%$ carbohydrate, $30 \%$ fat), which has been shown to produce significantly lower blood pressure and glycemic responses. ${ }^{99,107}$ Asemi and Esmaillzadeh ${ }^{78}$ demonstrated that adherence to DASH eating pattern, compared to a control diet based on Iranian traditional foods, resulted in greater reductions in body weight, $\mathrm{BMI}, \mathrm{WC}$, hip circumference (HC), serum insulin levels, IR, hsCRP and TG compared to the control diet in women with PCOS. ${ }^{106}$ Similarly, another trial showed that adherence to the DASH diet for 12 weeks had beneficial effects on weight loss, serum AMH, markers of insulin metabolism, SHBG, FAI, plasma nitric oxide and malondialdehyde levels among women with PCOS. ${ }^{105}$

In conclusion, replacement of dietary carbohydrate with MUFA and/or PUFA in a reduced energy diet may offer additional health benefits in the management of PCOS. The adoption of healthy dietary patterns such as DASH or the Mediterranean-style diets should be encouraged among women with PCOS, as they are rich in dietary fiber, antioxidants and anti-inflammatory nutrients, lead to greater satiety and have anti-hyperlipidemic, antihypertensive and antidiabetic properties. ${ }^{78,103,106}$

\section{PUFA and PCOS}

PUFAs, particularly the marine n-3 PUFA (eicosapentaenoic acid [EPA] and docosahexaenoic acid [DHA]), have been studied for their metabolic effects on insulin sensitivity, IR, androgen status and anti-inflammatory markers in women with PCOS. ${ }^{16,108-111}$ Mainly, most of the clinical trials of PUFA supplementation were of short duration (6-12 weeks) and the supplementation dose ranged between 2 and $4 \mathrm{~g}$ PUFAs per day. ${ }^{108-110,112-118}$ A recent meta-analysis of eight clinical trials with a total of 298 females with PCOS showed a slight reduction in serum total testosterone levels following n-3 PUFA supplementation among females with PCOS, without significant impact on SHBG and DHEAS levels. ${ }^{119}$ Results from a cross-sectional study showed that increased plasma n-6 PUFAs or increased n-6:n-3 PUFA ratio was associated with greater circulating androgen concentrations, whereas increased long-chain n-3 PUFA in plasma was associated with decreased blood lipids in women with PCOS. ${ }^{110}$ Subsequently, results from a crossover, placebo-controlled, RCT showed that long-chain n-3 PUFA supplementation $(4 \times 1 \mathrm{~g}$ capsules/ day, containing $2.4 \mathrm{~g}$ total long-chain n-3 PUFAs, $1.9 \mathrm{~g}$ EPA and DHA/day in a ratio of EPA to DHA of 1.49:1) resulted in increased plasma concentrations of n-3 PUFAs and had an antiandrogenic effect in women with PCOS. ${ }^{110}$ Results from a double-blind RCT, providing n-3 PUFA supplementation ( $3 \mathrm{~g} /$ day) for 8 weeks, showed that n-3 PUFA led to a greater reduction in serum testosterone, but not $\mathrm{SHBG}$, compared to the placebo group. ${ }^{112}$ Results from another double-blind RCT, providing n-3 PUFA supplementation ( $2 \mathrm{~g}$ /day) for 6 months in PCOS, showed that n-3 decreased lipid profiles, WC and interval between periods, with no changes in body weight, $\mathrm{HC}$, fasting glucose, number of ovarian follicles, size of ovary, bleeding volume, menstrual bleeding and hirsutism score. ${ }^{120}$

Some have proposed that n-3 PUFAs may improve insulin sensitivity by decreasing the production of inflammatory cytokines including tumor necrosis factor alpha (TNF- $\alpha$ ), interleukin 6 (IL-6) and increasing secretion of the anti-inflammatory hormone adiponectin. ${ }^{121}$ However, results from clinical trials on IR and insulin sensitivity remain controversial in women with PCOS. Nasri et $a^{113}$ reported that $2 \mathrm{~g}$ n-3 PUFA supplementation from flaxseed oil (800 $\mathrm{mg} \alpha$-linolenic acid [ALA]/ day) had beneficial effects on peroxisome proliferator-activated receptor gamma (PPAR- $\gamma$ ) and LDL receptor gene expression involved in the insulin and lipid signaling pathways. Similarly, Mohammadi et al ${ }^{114}$ showed that $4 \mathrm{~g}$ n-3 PUFA supplementation (720 mg EPA and $480 \mathrm{mg}$ DHA) resulted in a $20 \%$ increase in adiponectin and decreased glucose $(-11 \%)$ and insulin $(-8 \%)$ concentrations. However, a recent meta-analysis with three available trials of small sample size reported no beneficial effects of n-3 PUFAs on IR or other clinical outcome measures in women with PCOS. ${ }^{121}$

In conclusion, although some studies have reported some beneficial health effects of PUFAS, particularly n-3 marine PUFAs, there is still a lot of controversy and no conclusions can be drawn at this point.

\section{Meal frequency, meal timing and PCOS}

Meal frequency and meal timing seem to be valuable components of lifestyle changes, although there are only limited 
data on women with PCOS. Some argue that frequent meal consumption is detrimental to body composition and indices of glycemic control, because it may lead to weight gain due to increased lipogenesis or fat deposition after meals or by simply increasing the overall energy intake. ${ }^{122-125}$ Furthermore, it has been suggested that increased meal frequency may increase postprandial glucose, insulin, IR and blood lipids and may have a negative impact on the fatty acid composition of serum phospholipids. ${ }^{126-129}$ In contrast, others propose that increased meal frequency may exert a beneficial effect on body weight and indices of glycemic control, which may be attributed to nutrient load spreading, producing lower postprandial insulin concentrations, hunger reduction, inhibitory effects of free fatty acids on glucose uptake suppression and increased glucose clearance from the circulation with a significant economy in insulin secretion. ${ }^{130,131}$

In a 24-week crossover RCT, our group reported a beneficial effect of a six-meal pattern, without energy restriction, on amelioration of post-oral glucose tolerance test (OGTT) insulin sensitivity and reduction in subjective hunger in lean and obese women with PCOS compared to a three-meal pattern. ${ }^{132}$ Another study by Jakubowicz et al ${ }^{133}$ compared two isocaloric diets (high-kilocalorie breakfast vs highkilocalorie dinner) in lean women with PCOS and reported that high-energy intake at breakfast resulted in reduced food intake at dinner, which in turn led to improved insulin sensitivity and markers of reproductive function.

The timing of food intake has gained considerable interest in the past few years as it is found to affect metabolism and insulin secretion. ${ }^{134}$ It has been suggested that postprandial glycemia is under circadian regulation and that its misalignment may lead to glucose intolerance. ${ }^{130}$ Eating late during the day was associated with decreased resting-energy expenditure, decreased fasting carbohydrate oxidation, decreased glucose tolerance, blunted daily profile in free cortisol concentrations and decreased thermal effect of food on wrist temperature in normal weight, healthy females. ${ }^{135}$ People with prediabetes preferring to consume their main meal in the evening (later chronotype) had higher HbAlc compared to those consuming their main course at lunch time, and thus, had higher risk of developing type 2 diabetes sooner. ${ }^{136}$ The American Heart Association in their recent scientific statement concluded that meal frequency and timing may be important parameters in the nutrition management of chronic diseases, leading to healthier lifestyle and reduction in cardiometabolic risk factors. ${ }^{128}$

\section{Vitamin D and PCOS}

Epidemiological studies have shown inconsistent results for vitamin D status between women with and without PCOS. ${ }^{137}$ Some studies have demonstrated lower serum vitamin D concentrations in women with PCOS compared to women without PCOS, whereas a case-control study, with 85 women with PCOS and 115 healthy controls, found higher serum vitamin D concentrations in women with PCOS compared to controls. ${ }^{138-140}$ Low serum vitamin D levels or insufficiency has been positively associated with PCOS-related symptoms, such as central obesity, IR, infertility and hirsutism, whereas serum 25-hydroxy vitamin D (25OHD) seems to be an independent predictor of measures of reproductive success following ovulation induction. ${ }^{141-144}$

A recent systematic review and meta-analysis showed that vitamin D supplementation significantly decreased total testosterone, without any effects on serum SHBG and free testosterone. ${ }^{137}$ Another systematic review discussed the association between vitamin $\mathrm{D}$ status and metabolic disturbances and suggested an inverse association between vitamin $\mathrm{D}$ status and IR in women with PCOS. ${ }^{145}$ One study showed that $1000 \mathrm{mg} /$ day calcium plus 50,000 IU/week vitamin D supplementation for 8 weeks among vitamin D-deficient women with PCOS had beneficial effects on serum insulin levels, IR, TG and very-LDL (VLDL) levels, without affecting fasting glucose and other lipid profiles. ${ }^{146}$ Another study showed that the combination of $1500 \mathrm{mg}$ /day metformin plus $1000 \mathrm{mg} /$ day calcium and 100,000 IU/month vitamin D supplementation for 6 months had positive effects on weight loss, follicle maturation, menstrual regularity and improvement in hyperandrogenism in infertile women with PCOS. ${ }^{147}$

In conclusion, few available studies have found some, but limited, benefits of vitamin D supplementation among women with PCOS, and it remains a controversial topic. Systematic reviews and meta-analyses support that currently no recommendations can be made about vitamin D supplementation for PCOS, due to conflicting study results, small sample sizes, lack of adjustments for confounders, use of different definitions for PCOS, use of different assays for serum 25OHD measurement, short trial duration and use of varying amounts of vitamin D supplementation. ${ }^{145}$

\section{PCOS and micronutrients}

Few available studies have examined the effects of micronutrient supplementation on PCOS symptoms and biochemical indices. One study showed that $220 \mathrm{mg}$ zinc sulfate supplementation per day for 8 weeks among PCOS had beneficial 
effects on fasting glucose, insulin, IR and TG. ${ }^{148}$ It has been reported that $200 \mathrm{mg} /$ day selenium supplementation for 8 weeks among PCOS had beneficial effects on fasting insulin, IR, insulin sensitivity, TG and VLDL levels, without affecting fasting glucose and other lipid profiles. ${ }^{149}$ Another study found that $200 \mathrm{mg}$ /day chromium supplementation for 8 weeks among PCOS had favorable effects on fasting insulin, IR and insulin sensitivity. ${ }^{150}$ Moreover, $5 \mathrm{mg}$ /day folate supplementation for 8 weeks among PCOS had beneficial effects on inflammatory factors and biomarkers of oxidative stress. ${ }^{151}$ In conclusion, there is insufficient evidence to recommend micronutrient supplements for women with PCOS at this time.

In summary, this review aimed to discuss the effects of nutritional support and dietary interventions on various clinical outcome measures in women with PCOS. The results indicate that there is still a great need for well-designed, long-term, with adequate sample size, clinical trials examining all aspects of overall nutrition (ie, patterns, habits and alternative nutrition interventions) and nutrient effects (ie, micronutrients) in women with PCOS. From the available current trial data, we can conclude that LSM, including a small-to-moderate weight loss of about $7 \%$ achieved with any dietary pattern of choice, depending on the individuals' preferences, culture, habits and metabolic needs (ie, DASH diet, moderately low-carbohydrate diets [ $40-45 \%$ of energy] or substitution of carbohydrates for MUFA or PUFA in the diet), as well as alternative dietary interventions, including increased meal frequency and regular meal timing, with the majority of carbohydrates consumed at lunch time, seems to offer the evidence-based nutritional support strategy for IR and the management and treatment of PCOS.

\section{Disclosure}

The authors report no conflicts of interest in this work.

\section{References}

1. Bergh CM, Moore M, Gundell C. Evidence-based management of infertility in women with polycystic ovary syndrome. J Obstet Gynecol Neonatal Nurs. 2016;45(1):111-122.

2. Moran LJ, Hutchison SK, Norman RJ, Teede HJ. Lifestyle changes in women with polycystic ovary syndrome. Wiley. 2011.

3. Group TREA-SPCW. Revised 2003 consensus on diagnostic criteria and long-term health risks related to polycystic ovary syndrome. Fertil Steril. 2004;81(1):19-25.

4. Azziz R, Carmina E, Dewailly D, et al. The Androgen Excess and PCOS Society criteria for the polycystic ovary syndrome: the complete task force report. Fertil Steril. 2009;91(2):456-488.

5. Messinis IE, Messini CI, Anifandis G, Dafopoulos K. Polycystic ovaries and obesity. Best Pract Res Clin Obstet Gynaecol. 2015;29(4):479-488.

6. Kjerulff LE, Sanchez-Ramos L, Duffy D. Pregnancy outcomes in women with polycystic ovary syndrome: a metaanalysis. Am J Obstet Gynecol. 2011;204(6):558.e1-558.e6.
7. Sterling L, Liu J, Okun N, Sakhuja A, Sierra S, Greenblatt E. Pregnancy outcomes in women with polycystic ovary syndrome undergoing in vitro fertilization. Fertil Steril. 2016;105(3):791.e2-797.e2.

8. Palomba S, Santagni S, Falbo A, La Sala GB. Complications and challenges associated with polycystic ovary syndrome: current perspectives. Int J Womens Health. 2015;7:745-763.

9. Manco M, Castagneto-Gissey L, Arrighi E, et al. Insulin dynamics in young women with polycystic ovary syndrome and normal glucose tolerance across categories of body mass index. PLoS One. 2014;9(4):e92995.

10. Trakakis E, Basios G, Peppa M, et al. The prevalence of glucose metabolism abnormalities in Greek women with polycystic ovary syndrome. Gynecol Endocrinol. 2012;28(11):867-870.

11. Dunaif A. Insulin resistance and the polycystic ovary syndrome: mechanism and implications for pathogenesis. Endocr Rev. 1997;18(6):774-800.

12. Goodarzi MO, Dumesic DA, Chazenbalk G, Azziz R. Polycystic ovary syndrome: etiology, pathogenesis and diagnosis. Nat Rev Endocrinol. 2011;7(4):219-231.

13. Azziz R, Carmina E, Chen Z, et al. Polycystic ovary syndrome. Nat Rev Dis Primers. 2016;2:16057.

14. DeUgarte CM, Bartolucci AA, Azziz R. Prevalence of insulin resistance in the polycystic ovary syndrome using the homeostasis model assessment. Fertil Steril. 2005;83(5):1454-1460.

15. Norman RJ, Davies M, Lord J, Moran LJ. The role of lifestyle modification in polycystic ovary syndrome. Trends Endocrinol Metab. 2002;13(6):251-257.

16. Rondanelli M, Perna S, Faliva M, Monteferrario F, Repaci E, Allieri F. Focus on metabolic and nutritional correlates of polycystic ovary syndrome and update on nutritional management of these critical phenomena. Arch Gynecol Obstet. 2014;290(6):1079-1092.

17. Cassar S, Misso ML, Hopkins WG, Shaw CS, Teede HJ, Stepto NK. Insulin resistance in polycystic ovary syndrome: a systematic review and meta-analysis of euglycaemic-hyperinsulinaemic clamp studies. Hum Reprod. 2016;31(11):2619-2631.

18. Song DK, Hong YS, Sung YA, Lee H. Insulin resistance according to beta-cell function in women with polycystic ovary syndrome and normal glucose tolerance. PLoS One. 2017;12(5):e0178120.

19. Glueck CJ, Dharashivkar S, Wang P, et al. Obesity and extreme obesity, manifest by ages 20-24 years, continuing through 32-41 years in women, should alert physicians to the diagnostic likelihood of polycystic ovary syndrome as a reversible underlying endocrinopathy. Eur J Obstet Gynecol Reprod Biol. 2005;122(2):206-212.

20. Graff SK, Mario FM, Alves BC, Spritzer PM. Dietary glycemic index is associated with less favorable anthropometric and metabolic profiles in polycystic ovary syndrome women with different phenotypes. Fertil Steril. 2013;100(4):1081-1088.

21. Larsson I, Hulthen L, Landen M, Palsson E, Janson P, Stener-Victorin E. Dietary intake, resting energy expenditure, and eating behavior in women with and without polycystic ovary syndrome. Clin Nutr. 2016;35(1):213-218

22. Ollila ME, West S, Keinanen-Kiukaanniemi S, et al. Overweight and obese but not normal weight women with PCOS are at increased risk of Type 2 diabetes mellitus-a prospective, population-based cohort study. Hum Reprod. 2017;32(2):423-431.

23. Stankiewicz Marcin NR. Diagnosis and management of polycystic ovary syndrome: a practical guide. Drugs. 2006;66(7):903-912.

24. Holte J, Bergh T, Berne C, Lithell H. Serum lipoprotein lipid profile in women with the polycystic ovary syndrome: relation to anthropometric, endocrine and metabolic variables. Clin Endocrinol (Oxf). 1994;41(4):463-471.

25. Rodríguez-Morán M, Guerrero-Romero F. Insulin resistance is independently related to age in Mexican women. $J$ Endocrinol Invest. 2003;26:42-48.

26. Stepto NK, Cassar S, Joham AE, et al. Women with polycystic ovary syndrome have intrinsic insulin resistance on euglycaemic-hyperinsulaemic clamp. Hum Reprod. 2013;28(3):777-784. 
27. Liou TH, Yang JH, Hsieh CH, Lee CY, Hsu CS, Hsu MI. Clinical and biochemical presentations of polycystic ovary syndrome among obese and nonobese women. Fertil Steril. 2009;92(6):1960-1965.

28. Brassard M, AinMelk Y, Baillargeon JP. Basic infertility including polycystic ovary syndrome. Med Clin North Am. 2008;92(5):11631192 , xi.

29. Lim SS, Norman RJ, Davies MJ, Moran LJ. The effect of obesity on polycystic ovary syndrome: a systematic review and meta-analysis. Obes Rev. 2013;14(2):95-109.

30. Brewer CJ, Balen AH. The adverse effects of obesity on conception and implantation. Reproduction. 2010;140(3):347-364.

31. de Groot PC, Dekkers OM, Romijn JA, Dieben SW, Helmerhorst FM. PCOS, coronary heart disease, stroke and the influence of obesity: a systematic review and meta-analysis. Hum Reprod Update. 2011;17(4):495-500

32. Hudecova M, Holte J, Olovsson M, Larsson A, Berne C, SundstromPoromaa I. Prevalence of the metabolic syndrome in women with a previous diagnosis of polycystic ovary syndrome: long-term follow-up. Fertil Steril. 2011;96(5):1271-1274.

33. Moran LJ, Misso ML, Wild RA, Norman RJ. Impaired glucose tolerance, type 2 diabetes and metabolic syndrome in polycystic ovary syndrome: a systematic review and meta-analysis. Hum Reprod Update. 2010;16(4):347-363.

34. Jalilian N, Haghnazari L, Rasolinia S. Leptin and body mass index in polycystic ovary syndrome. Indian J Endocrinol Metab. 2016;20(3):324-328.

35. Lin AW, Lujan ME. Comparison of dietary intake and physical activity between women with and without polycystic ovary syndrome: a review. Adv Nutr. 2014;5(5):486-496.

36. Moran LJ, Grieger JA, Mishra GD, Teede HJ. The association of a Mediterranean-style diet pattern with polycystic ovary syndrome status in a community cohort study. Nutrients. 2015;7(10):8553-8564.

37. Turner-McGrievy G, Davidson CR, Billings DL. Dietary intake, eating behaviors, and quality of life in women with polycystic ovary syndrome who are trying to conceive. Hum Fertil. 2015;18(1):16-21.

38. Moran LJ, Pasquali R, Teede HJ, Hoeger KM, Norman RJ. Treatment of obesity in polycystic ovary syndrome: a position statement of the Androgen Excess and Polycystic Ovary Syndrome Society. Fertil Steril. 2009;92(6):1966-1982.

39. Salley KE, Wickham EP, Cheang KI, Essah PA, Karjane NW, Nestler JE. Glucose intolerance in polycystic ovary syndrome - a position statement of the Androgen Excess Society. J Clin Endocrinol Metab. 2007;92(12):4546-4556.

40. Bates GW, Legro RS. Longterm management of polycystic ovarian syndrome (PCOS). Mol Cell Endocrinol. 2013;373(1-2):91-97.

41. Moran LJ, Noakes M, Clifton PM, Tomlinson L, Galletly C, Norman RJ. Dietary composition in restoring reproductive and metabolic physiology in overweight women with polycystic ovary syndrome. J Clin Endocrinol Metab. 2003;88(2):812-819.

42. Stamets K, Taylor DS, Kunselman A, Demers LM, Pelkman CL, Legro RS. A randomized trial of the effects of two types of short-term hypocaloric diets on weight loss in women with polycystic ovary syndrome. Fertil Steril. 2004;81(3):630-637.

43. Tolino A, Gambardella V, Caccavale C, et al. Evaluation of ovarian functionality after a dietary treatment in obese women with polycystic ovary syndrome. Eur J Obstet Gynecol Reprod Biol. 2005;119(1):87-93.

44. Frary JMC, Bjerre KP, Glintborg D, Ravn P. The effect of dietary carbohydrates in women with polycystic ovary syndrome. A systematic review. Minerva Endocrinol. 2016;41(1):57-69.

45. Badawy A, Elnashar A. Treatment options for polycystic ovary syndrome. Int $J$ Womens Health. 2011;3:25-35.

46. Glintborg D, Petersen MH, Ravn P, Hermann AP, Andersen M. Comparison of regional fat mass measurement by whole body DXA scans and anthropometric measures to predict insulin resistance in women with polycystic ovary syndrome and controls. Acta Obstet Gynecol Scand. 2016;95(11):1235-1243.
47. Thessaloniki ESHRE/ASRM-Sponsored PCOS Consensus Workshop Group. Consensus on infertility treatment related to polycystic ovary syndrome. Fertil Steril. 2008;89(3):505-522.

48. El Hayek S, Bitar L, Hamdar LH, Mirza FG, Daoud G. Poly cystic ovarian syndrome: an updated overview. Front Physiol. 2016;7:124.

49. Mahalingaiah S, Diamanti-Kandarakis E. Targets to treat metabolic syndrome in polycystic ovary syndrome. Expert Opin Ther Targets. 2015;19(11):1561-1574.

50. Legro RS. Ovulation induction in polycystic ovary syndrome: current options. Best Pract Res Clin Obstet Gynaecol. 2016;37:152-159.

51. Thessaloniki ESHRE/ASRM-Sponsored PCOS Consensus Workshop Group. Consensus on infertility treatment related to polycystic ovary syndrome. Hum Reprod. 2008;23(3):462-477.

52. Palomba S, Falbo A, Giallauria F, et al. Six weeks of structured exercise training and hypocaloric diet increases the probability of ovulation after clomiphene citrate in overweight and obese patients with polycystic ovary syndrome: a randomized controlled trial. Hum Reprod. 2010;25(11):2783-2791.

53. Legro RS, Dodson WC, Kunselman AR, et al. Benefit of delayed fertility therapy with preconception weight loss over immediate therapy in obese women with PCOS. J Clin Endocrinol Metab. 2016;101(7):2658-2666.

54. Legro RS, Dodson WC, Kris-Etherton PM, et al. Randomized controlled trial of preconception interventions in infertile women with polycystic ovary syndrome. J Clin Endocrinol Metab. 2015;100(11): 4048-4058.

55. Franik S, Kremer JA, Nelen WL, Farquhar C, Marjoribanks J. Aromatase inhibitors for subfertile women with polycystic ovary syndrome: summary of a Cochrane review. Fertil Steril. 2015;103(2):353-355.

56. Yu Y, Fang L, Zhang R, et al. Comparative effectiveness of 9 ovulation-induction therapies in patients with clomiphene citrateresistant polycystic ovary syndrome: a network meta-analysis. Sci Rep. 2017;7(1):3812.

57. Naderpoor N, Shorakae S, de Courten B, Misso ML, Moran LJ, Teede HJ. Metformin and lifestyle modification in polycystic ovary syndrome: systematic review and meta-analysis. Hum Reprod Update. 2015;21(5):560-574.

58. Balen AH, Morley LC, Misso M, et al. The management of anovulatory infertility in women with polycystic ovary syndrome: an analysis of the evidence to support the development of global WHO guidance. Hum Reprod Update. 2016;22(6):687-708.

59. Hwang YI, Cha SW, Song IO, Yang KM, Min EG, Kim HO. Fertility of patients with polycystic ovary syndrome undergoing in vitro fertilization by age. Int J Gynaecol Obstet. 2016;135(1):91-95.

60. Scoccia B. What is new in polycystic ovary syndrome? Best articles from the past year. Obstet Gynecol. 2016;128(5):1174-1176.

61. Ujvari D, Hulchiy M, Calaby A, Nybacka A, Bystrom B, Hirschberg AL. Lifestyle intervention up-regulates gene and protein levels of molecules involved in insulin signaling in the endometrium of overweight/obese women with polycystic ovary syndrome. Hum Reprod. 2014;29(7):1526-1535.

62. Mahoney D. Lifestyle modification intervention among infertile overweight and obese women with polycystic ovary syndrome. $J$ Am Assoc Nurse Pract. 2014;26(6):301-308.

63. Haqq L, McFarlane J, Dieberg G, Smart N. Effect of lifestyle intervention on the reproductive endocrine profile in women with polycystic ovarian syndrome: a systematic review and meta-analysis. Endocr Connect. 2014;3(1):36-46.

64. Jarrett BY, Lujan ME. Impact of hypocaloric dietary intervention on ovulation in obese women with PCOS. Reproduction. Epub 2016 Oct 31 .

65. Moran LJ, Brown WJ, McNaughton SA, Joham AE, Teede HJ. Weight management practices associated with PCOS and their relationships with diet and physical activity. Hum Reprod. 2017;32(3):669-678.

66. Sharma A, Walker DM, Atiomo W. National survey on management of weight reduction in PCOS women in the United Kingdom. Eur $J$ Obstet Gynecol Reprod Biol. 2010;152(2):181-185. 
67. Gibson-Helm ME, Lucas IM, Boyle JA, Teede HJ. Women's experiences of polycystic ovary syndrome diagnosis. Fam Pract. 2014;31(5):545-549.

68. Dalle Grave R, Calugi S, El Ghoch M. Lifestyle modification in the management of obesity: achievements and challenges. Eat Weight Disord. 2013;18(4):339-349.

69. Gillies CL, Abrams KR, Lambert PC, et al. Pharmacological and lifestyle interventions to prevent or delay type 2 diabetes in people with impaired glucose tolerance: systematic review and meta-analysis. BMJ. 2007;334(7588):299.

70. Domecq JP, Prutsky G, Mullan RJ, et al. Lifestyle modification programs in polycystic ovary syndrome: systematic review and metaanalysis. J Clin Endocrinol Metab. 2013;98(12):4655-4663.

71. Moran LJ, Hutchison SK, Norman RJ, Teede HJ. Lifestyle changes in women with polycystic ovary syndrome. Cochrane Database Syst Rev. 2011;7:CD007506.

72. Haqq L, McFarlane J, Dieberg G, Smart N. The effect of lifestyle intervention on body composition, glycemic control, and cardiorespiratory fitness in polycystic ovarian syndrome: a systematic review and meta-analysis. Int J Sport Nutr Exerc Metab. 2015;25(6):533-540.

73. Hoeger KM, Kochman L, Wixom N, Craig K, Miller RK, Guzick DS. A randomized, 48-week, placebo-controlled trial of intensive lifestyle modification and/or metformin therapy in overweight women with polycystic ovary syndrome: a pilot study. Fertil Steril. 2004;82(2):421-429.

74. Montesi L, El Ghoch M, Brodosi L, Calugi S, Marchesini G, Dalle Grave R. Long-term weight loss maintenance for obesity: a multidisciplinary approach. Diabetes Metab Syndr Obes. 2016;9:37-46.

75. Dalle Grave R, Calugi S, Centis E, Marzocchi R, El Ghoch M, Marchesini G. Lifestyle modification in the management of the metabolic syndrome: achievements and challenges. Diabetes Metab Syndr Obes. 2010;3:373-385.

76. Goss AM, Chandler-Laney PC, Ovalle F, et al. Effects of a eucaloric reduced-carbohydrate diet on body composition and fat distribution in women with PCOS. Metabolism. 2014;63(10):1257-1264.

77. Marzouk TM, Sayed Ahmed WA. Effect of dietary weight loss on menstrual regularity in obese young adult women with polycystic ovary syndrome. J Pediatr Adolesc Gynecol. 2015;28(6):457-461.

78. Asemi Z, Esmaillzadeh A. DASH diet, insulin resistance, and serum hs-CRP in polycystic ovary syndrome: a randomized controlled clinical trial. Horm Metab Res. 2015;47(3):232-238.

79. Marsh KA, Steinbeck KS, Atkinson FS, Petocz P, Brand-Miller JC. Effect of a low glycemic index compared with a conventional healthy diet on polycystic ovary syndrome. Am J Clin Nutr. 2010;92(1): 83-92.

80. Moran LJ, Ko H, Misso M, et al. Dietary composition in the treatment of polycystic ovary syndrome: a systematic review to inform evidencebased guidelines. J Acad Nutr Diet. 2013;113(4):520-545.

81. Moran LJ, Noakes M, Clifton PM, Wittert GA, Williams G, Norman RJ. Short-term meal replacements followed by dietary macronutrient restriction enhance weight loss in polycystic ovary syndrome. Am J Clin Nutr. 2006;2006(84):77-87.

82. Soares NP, Santos AC, Costa EC, et al. Diet-induced weight loss reduces DNA damage and cardiometabolic risk factors in overweight/ obese women with polycystic ovary syndrome. Ann Nutr Metab. 2016;68(3):220-227.

83. Mehrabani HH, Salehpour S, Amiri Z, Farahani SJ, Meyer BJ, Tahbaz F. Beneficial effects of a high-protein, low-glycemic-load hypocaloric diet in overweight and obese women with polycystic ovary syndrome: a randomized controlled intervention study. J Am Coll Nutr. 2012;31(2):117-125.

84. Freedman MR, King J, Kennedy E. Popular diets: a scientific review. Obes Res. 2001;9(1).

85. Mavropoulos JC, Yancy WS, Hepburn J, Westman EC. The effects of a low-carbohydrate, ketogenic diet on the polycystic ovary syndrome: a pilot study. Nutr Metab (Lond). 2005;2:35.
86. Wong JM, Gallagher M, Gooding H, et al. A randomized pilot study of dietary treatments for polycystic ovary syndrome in adolescents. Pediatr Obes. 2015;11(3):210-220.

87. Sørensen LB, Soe M, Halkier KH, Stigsby B, Astrup A. Effects of increased dietary protein-to-carbohydrate ratios in women with polycystic ovary syndrome. Am J Clin Nutr. 2012;95(1):39-48.

88. Toscani MK, Mario FM, Radavelli-Bagatini S, Wiltgen D, Matos MC, Spritzer PM. Effect of high-protein or normal-protein diet on weight loss, body composition, hormone, and metabolic profile in southern Brazilian women with polycystic ovary syndrome: a randomized study. Gynecol Endocrinol. 2011;27(11):925-930.

89. Kasim-Karakas SE, Almario RU, Cunningham W. Effects of protein versus simple sugar intake on weight loss in polycystic ovary syndrome (according to the National Institutes of Health criteria). Fertil Steril. 2009;92(1):262-270.

90. Perelman D, Coghlan N, Lamendola C, Carter S, Abbasi F, McLaughlin T. Substituting poly- and mono-unsaturated fat for dietary carbohydrate reduces hyperinsulinemia in women with polycystic ovary syndrome. Gynecol Endocrinol. 2017;33(4):324-327.

91. Gower BA, Chandler-Laney PC, Ovalle F, et al. Favourable metabolic effects of a eucaloric lower-carbohydrate diet in women with PCOS. Clin Endocrinol (Oxf). 2013;79(4):550-557.

92. Douglas CC, Gower BA, Darnell BE, Ovalle F, Oster RA, Azziz R. Role of diet in the treatment of polycystic ovary syndrome. Fertil Steril. 2006;85(3):679-688.

93. Pearce KL, Noakes M, Keogh J, Clifton PM. Effect of carbohydrate distribution on postprandial glucose peaks with the use of continuous glucose monitoring in type 2 diabetes. Am J Clin Nutr. 2008;87:638-644.

94. Kang X, Wang C, Lifang L, et al. Effects of different proportion of carbohydrate in breakfast on postprandial glucose excursion in normal glucose tolerance and impaired glucose regulation subjects. Diabetes Technol Ther. 2013;15(7):569-574.

95. Barr S, Reeves S, Sharp K, Jeanes YM. An isocaloric low glycemic index diet improves insulin sensitivity in women with polycystic ovary syndrome. J Acad Nutr Diet. 2013;113(11):1523-1531.

96. Phy JL, Pohlmeier AM, Cooper JA, et al. Low starch/low dairy diet results in successful treatment of obesity and co-morbidities linked to polycystic ovary syndrome (PCOS). J Obes Weight Loss Ther. 2015;5(2):259.

97. Dong JY, Zhang ZL, Wang PY, Qin LQ. Effects of high-protein diets on body weight, glycaemic control, blood lipids and blood pressure in type 2 diabetes: meta-analysis of randomised controlled trials. $\mathrm{Br}$ J Nutr. 2013;110(5):781-789.

98. Papakonstantinou E, Triantafillidou D, Panagiotakos DB, Iraklianou $\mathrm{S}$, Berdanier CD, Zampelas A. A high protein low fat meal does not influence glucose and insulin responses in obese individuals with or without type 2 diabetes. J Hum Nutr Diet. 2010;23(2):183-189.

99. American Diabetes Association. Standards of medical care in diabetes - 2017;40(Suppl 1).

100. Bray GA, Smith SR, de Jonge L, et al. Effect of dietary protein content on weight gain, energy expenditure, and body composition during overeating: a randomized controlled trial. JAMA. 2012;307(1):47-55.

101. Leidy HJ, Bossingham MJ, Mattes RD, Campbell WW. Increased dietary protein consumed at breakfast leads to an initial and sustained feeling of fullness during energy restriction compared to other meal times. Br J Nutr. 2008;101(06):798.

102. Koppes LL, Boon N, Nooyens AC, van Mechelen W, Saris WH. Macronutrient distribution over a period of 23 years in relation to energy intake and body fatness. Br J Nutr. 2009;101(1):108-115.

103. de la Iglesia R, Loria-Kohen V, Zulet MA, Martinez JA, Reglero G, Ramirez de Molina A. Dietary strategies implicated in the prevention and treatment of metabolic syndrome. Int J Mol Sci. 2016;17(11):E1877.

104. Bosse DJ, Dixon MB. Dietary protein in weight management: a review proposing protein spread and change theories. Nutr Metab. 2012;9(1):81. 
105. Foroozanfard F, Rafiei H, Samimi M, et al. The effects of dietary approaches to stop hypertension diet on weight loss, anti-Mullerian hormone and metabolic profiles in women with polycystic ovary syndrome: a randomized clinical trial. Clin Endocrinol (Oxf). 2017.

106. Asemi Z, Samimi M, Tabassi Z, Shakeri H, Sabihi SS, Esmaillzadeh A. Effects of DASH diet on lipid profiles and biomarkers of oxidative stress in overweight and obese women with polycystic ovary syndrome: a randomized clinical trial. Nutrition. 2014;30(11-12):1287-1293.

107. Vollmer WMSF, Ard J, Appel LJ, et al. Effects of diet and sodium intake on blood pressure: subgroup analysis of the DASH-sodium trial. Ann Intern Med. 2001;135:1019-1028.

108. Kalgaonkar S, Almario RU, Gurusinghe D, et al. Differential effects of walnuts vs almonds on improving metabolic and endocrine parameters in PCOS. Eur J Clin Nutr. 2011;65(3):386-393.

109. Karakas SE, Perroud B, Kind T, Palazoglu M, Fiehn O. Changes in plasma metabolites and glucose homeostasis during omega-3 polyunsaturated fatty acid supplementation in women with polycystic ovary syndrome. BBA Clin. 2016;5:179-185.

110. Phelan N, O'Connor A, Kyaw Tun T, et al. Hormonal and metabolic effects of polyunsaturated fatty acids in young women with polycystic ovary syndrome: results from a cross-sectional analysis and a randomized, placebo-controlled, crossover trial. Am J Clin Nutr. 2011;93(3):652-662.

111. Macut D, Bjekic-Macut J, Savic-Radojevic A. Dyslipidemia and oxidative stress in PCOS. Front Horm Res. 2013;40:51-63.

112. Nadjarzadeh A, Firouzabadi RD, Daneshbodi NV, Lotfi MH, MozaffariKhosravi H. The effect of omega-3 supplementation on androgen profile and menstrual status in women with polycystic ovary syndrome: a randomized clinical trial. Iran J Reprod Med. 2013;11(8): 665-672.

113. Nasri K, Hantoushzadeh S, Aghadavod E, Taghizadeh M, Asemi Z. The effects of omega-3 fatty acids supplementation on gene expression involved in the insulin and lipid signaling pathway in patients with polycystic ovary syndrome. Horm Metab Res. 2017;49(6):446-451.

114. Mohammadi E, Rafraf M, Farzadi L, Asghari-Jafarabadi M, Sabour S. Effects of omega-3 fatty acids supplementation on serum adiponectin levels and some metabolic risk factors in women with polycystic ovary syndrome. Asia Pac J Clin Nutr. 2012;21(4):511-518.

115. Oner G, Muderris II. Efficacy of omega-3 in the treatment of polycystic ovary syndrome. J Obstet Gynaecol. 2013;33(3):289-291.

116. Vargas ML, Almario RU, Buchan W, Kim K, Karakas SE. Metabolic and endocrine effects of long-chain versus essential omega-3 polyunsaturated fatty acids in polycystic ovary syndrome. Metabolism. 2011;60(12):1711-1718.

117. Cussons AJ, Watts GF, Mori TA, Stuckey BG. Omega-3 fatty acid supplementation decreases liver fat content in polycystic ovary syndrome: a randomized controlled trial employing proton magnetic resonance spectroscopy. J Clin Endocrinol Metab. 2009;94(10):3842-3848.

118. Kasim-Karakas SE, Almario RU, Gregory L, Wong R, Todd H, Lasley BL. Metabolic and endocrine effects of a polyunsaturated fatty acidrich diet in polycystic ovary syndrome. J Clin Endocrinol Metab. 2004;89(2):615-620.

119. Hajishafiee M, Askari G, Iranj B, et al. The effect of n-3 polyunsaturated fatty acid supplementation on androgen status in patients with polycystic ovary syndrome: a systematic review and meta-analysis of clinical trials. Horm Metab Res. 2016;48(5):281-289.

120. Khani B, Mardanian F, Fesharaki SJ. Omega-3 supplementation effects on polycystic ovary syndrome symptoms and metabolic syndrome. J Res Med Sci. 2017;22:64.

121. Sadeghi A, Djafarian K, Mohammadi H, Shab-Bidar S. Effect of omega-3 fatty acids supplementation on insulin resistance in women with polycystic ovary syndrome: meta-analysis of randomized controlled trials. Diabetes Metab Syndr. 2016;11(2):157-162.

122. Howarth NC, Huang TT, Roberts SB, Lin BH, McCrory MA. Eating patterns and dietary composition in relation to BMI in younger and older adults. Int J Obes. 2007;31(4):675-684.
123. Duval K, Strychar I, Cyr MJ, Prud'homme D, Rabasa-Lhoret R, Doucet E. Physical activity is a confounding factor of the relation between eating frequency and body composition. Am J Clin Nutr. 2008;88(5):1200-1205.

124. Tai MM, Castillo P, Pi-Sunyer FX. Meal size and frequency: effect on the thermic effect of food. Am J Clin Nutr. 1991;54(5):783-787.

125. van der Heijden AA, Hu FB, Rimm EB, van Dam RM. A prospective study of breakfast consumption and weight gain among U.S. men. Obesity. 2007;15(10):2463-2469.

126. Mattson MP. The need for controlled studies of the effects of meal frequency on health. Lancet. 2005;365(9475):1978-1980.

127. Kahleova H, Belinova L, Malinska H, et al. Eating two larger meals a day (breakfast and lunch) is more effective than six smaller meals in a reduced-energy regimen for patients with type 2 diabetes: a randomised crossover study. Diabetologia. 2014;57(8):1552-1560.

128. St-Onge MP, Ard J, Baskin ML, et al. Meal timing and frequency: implications for cardiovascular disease prevention: a scientific statement from the American Heart Association. Circulation. 2017;135(9):e96-e121.

129. Holmstrup ME, Owens CM, Fairchild TJ, Kanaley JA. Effect of meal frequency on glucose and insulin excursions over the course of a day. Eur E J Clin Nutr Metab. 2010;5(6):e277-e280.

130. Kalsbeek A, la Fleur S, Fliers E. Circadian control of glucose metabolism. Mol Metab. 2014;3(4):372-383.

131. Jenkins D, Ocana A, Jenkins A, et al. Metabolic advantages of spreading the nutrient load: effects of increased meal frequency in non-insulin-dependent diabetes. Am J Clin Nutr. 1992;55(2):461-467.

132. Papakonstantinou E, Kechribari I, Mitrou P, et al. Effect of meal frequency on glucose and insulin levels in women with polycystic ovary syndrome: a randomised trial. Eur J Clin Nutr. 2016;70(5):588-594.

133. Jakubowicz D, Barnea M, Wainstein J, Froy O. Effects of caloric intake timing on insulin resistance and hyperandrogenism in lean women with polycystic ovary syndrome. Clin Sci (Lond). 2013;125(9):423-432.

134. Oike H, Oishi K, Kobori M. Nutrients, clock genes, and chrononutrition. Curr Nutr Rep. 2014;3:204-212.

135. Bandin C, Scheer FA, Luque AJ, et al. Meal timing affects glucose tolerance, substrate oxidation and circadian-related variables: a randomized, crossover trial. Int J Obes. 2015;39(5):828-833.

136. Anothaisintawee T, Lertrattananon D, Thamakaison S, Knutson KL, Thakkinstian A, Reutrakul S. Later chronotype is associated with higher hemoglobin A1c in prediabetes patients. Chronobiol Int. 2017;34(3):393-402.

137. Azadi-Yazdi M, Nadjarzadeh A, Khosravi-Boroujeni H, Salehi-Abargouei A. The effect of vitamin D supplementation on the androgenic profile in patients with polycystic ovary syndrome: a systematic review and meta-analysis of clinical trials. Horm Metab Res. 2017;49(3):174-179.

138. Mazloomi S, Sharifi F, Hajihosseini R, Kalantari S, Mazloomzadeh $\mathrm{S}$. Association between hypoadiponectinemia and low serum concentrations of calcium and vitamin D in women with polycystic ovary syndrome. ISRN Endocrinol. 2012;2012:949427.

139. Wehr E, Trummer O, Giuliani A, Gruber HJ, Pieber TR, ObermayerPietsch B. Vitamin D-associated polymorphisms are related to insulin resistance and vitamin D deficiency in polycystic ovary syndrome. Eur J Endocrinol. 2011;164(5):741-749.

140. Mahmoudi T, Gourabi H, Ashrafi M, Yazdi RS, Ezabadi Z. Calciotropic hormones, insulin resistance, and the polycystic ovary syndrome. Fertil Steril. 2010;93(4):1208-1214.

141. Thomson RL, Spedding S, Buckley JD. Vitamin D in the aetiology and management of polycystic ovary syndrome. Clin Endocrinol (Oxf). 2012;77(3):343-350.

142. Tzotzas T, Papadopoulou FG, Tziomalos K, et al. Rising serum 25-hydroxy-vitamin D levels after weight loss in obese women correlate with improvement in insulin resistance. J Clin Endocrinol Metab. 2010;95(9):4251-4257.

143. Joham AE, Teede HJ, Cassar S, et al. Vitamin D in polycystic ovary syndrome: relationship to obesity and insulin resistance. Mol Nutr Food Res. 2016;60(1):110-118. 
144. Pal L, Zhang H, Williams J, et al. Vitamin D status relates to reproductive outcome in women with polycystic ovary syndrome: secondary analysis of a multicenter randomized controlled trial. J Clin Endocrinol Metab. 2016;101(8):3027-3035.

145. Krul-Poel YH, Snackey C, Louwers Y, et al. The role of vitamin D in metabolic disturbances in polycystic ovary syndrome: a systematic review. Eur J Endocrinol. 2013;169(6):853-865.

146. Asemi Z, Foroozanfard F, Hashemi T, Bahmani F, Jamilian M, Esmaillzadeh A. Calcium plus vitamin D supplementation affects glucose metabolism and lipid concentrations in overweight and obese vitamin D deficient women with polycystic ovary syndrome. Clin Nutr. 2015;34(4):586-592.

147. Firouzabadi R, Aflatoonian A, Modarresi S, Sekhavat L, MohammadTaheri S. Therapeutic effects of calcium \& vitamin D supplementation in women with PCOS. Complement Ther Clin Pract. 2012;18(2):85-88.

148. Foroozanfard F, Jamilian M, Jafari Z, et al. Effects of zinc supplementation on markers of insulin resistance and lipid profiles in women with polycystic ovary syndrome: a randomized, double-blind, placebocontrolled trial. Exp Clin Endocrinol Diabetes. 2015;123(4):215-220.
149. Jamilian M, Razavi M, Fakhrie Kashan Z, Ghandi Y, Bagherian T, Asemi Z. Metabolic response to selenium supplementation in women with polycystic ovary syndrome: a randomized, double-blind, placebocontrolled trial. Clin Endocrinol. 2015;82(6):885-891.

150. Jamilian M, Asemi Z. Chromium supplementation and the effects on metabolic status in women with polycystic ovary syndrome: a randomized, double-blind, placebo-controlled trial. Ann Nutr Metab. 2015;67(1) 42-48.

151. Bahmani F, Karamali M, Shakeri H, Asemi Z. The effects of folate supplementation on inflammatory factors and biomarkers of oxidative stress in overweight and obese women with polycystic ovary syndrome: a randomized, double-blind, placebo-controlled clinical trial. Clin Endocrinol. 2014;81(4):582-587.

152. Turner-McGrievy GM, Davidson CR, Wingard EE, Billings DL. Low glycemic index vegan or low-calorie weight loss diets for women with polycystic ovary syndrome: a randomized controlled feasibility study. Nutr Res. 2014;34(6):552-558.

\section{Nutrition and Dietary Supplements}

\section{Publish your work in this journal}

Nutrition and Dietary Supplements is an international, peer-reviewed, open access journal focusing on research into nutritional requirements in health and disease, impact on metabolism and the identification and optimal use of dietary strategies and supplements necessary for normal growth and development. The journal welcomes submitted papers covering original research, basic science,

\section{Dovepress}

clinical \& epidemiological studies, reviews and evaluations, guidelines, expert opinion and commentary, case reports and extended reports. The manuscript management system is completely online and includes a very quick and fair peer-review system, which is all easy to use. Visit http://www.dovepress.com/ testimonials.php to read real quotes from published authors. 